\title{
Reachability Querying: An Independent Permutation Labeling Approach
}

\author{
Hao Wei, Jeffrey Xu Yu, Can Lu \\ Chinese University of Hong Kong \\ Hong Kong, China \\ \{hwei,yu,lucan\}@se.cuhk.edu.hk
}

\author{
Ruoming Jin \\ Kent State University \\ Kent, OH, USA \\ jin@cs.kent.edu
}

\begin{abstract}
Reachability query is a fundamental graph operation which answer$\mathrm{s}$ whether a vertex can reach another vertex over a large directed graph $G$ with $n$ vertices and $m$ edges, and has been extensively studied. In the literature, all the approaches compute a label for every vertex in a graph $G$ by index construction offline. The query time for answering reachability queries online is affected by the quality of the labels computed in index construction. The three main costs are the index construction time, the index size, and the query time. Some of the up-to-date approaches can answer reachability queries efficiently, but spend non-linear time to construct an index. Some of the up-to-date approaches construct an index in linear time and space, but may need to depth-first search $G$ at run-time in $O(n+m)$. In this paper, as the first, we propose a new randomized labeling approach to answer reachability queries, and the randomness is by independent permutation. We conduct extensive experimental studies to compare with the up-to-date approaches using 19 large real datasets used in the existing work and synthetic datasets. We confirm the efficiency of our approach.
\end{abstract}

\section{INTRODUCTION}

Reachability query is one of the fundamental graph operations to answer whether a vertex can reach another vertex over a large directed graph. The real applications that need this operation are many and can be found among online social networks, biological networks, ontology, transportation networks, etc. The reachability query has been extensively studied over a decade $[1,16,13,23,11$, $28,7,26,12,8,21,20,29,27,9,10,19,24,31]$, and the early work can be traced back to 1989 to compute transitive closure (TC) over a graph. However, it is still an unsolved question whether we can do faster to answer reachability queries online over even larger and/or even denser graphs with the possible minimum cost (time/space) for offline precomputing and preparation.

The main idea behind the approaches in the literature is to compute a label for every vertex in a graph $G$ by precomputing offline. This is known as index construction, because it is to construct an index to maintain all the labels computed to vertices of a graph. The index construction needs time and space to be done, and the

This work is licensed under the Creative Commons AttributionNonCommercial-NoDerivs 3.0 Unported License. To view a copy of this license, visit http://creativecommons.org/licenses/by-nc-nd/3.0/. Obtain permission prior to any use beyond those covered by the license. Contact copyright holder by emailing info@vldb.org. Articles from this volume were invited to present their results at the 40th International Conference on Very Large Data Bases, September 1st - 5th 2014, Hangzhou, China.

Proceedings of the VLDB Endowment, Vol. 7, No. 12

Copyright 2014 VLDB Endowment 2150-8097/14/08. quality of the labels computed offline will affect the query time for answering reachability queries online. In this work, we classify al1 the existing works in the literature into two categories. One is called Label-Only. By the name, the approaches in this category only use the labels computed to answer reachability queries, and include Chain-Cover [16, 8], Tree-Cover [1], Dual-Label [28], 2Hop [13], Path-Tree [21], 3-Hop [20], PWAH8 [27], TF-Label [10], $H L$ [19], and DL [19]. The other is called Label+G. By the name, the approaches in this category use labels computed where possible, and conduct depth-first-search (DFS) at run-time, if the reachability queries cannot be answered using the labels only. Such approaches include Tree+SSPI [7], GRIPP [26], GRAIL [29], and Ferrari [24].

All the approaches take a different way to balance the three main costs, namely, the index construction time, the index size, and the query time. The Label $+G$ approaches construct an index in linear (time/space). By linear we mean it in terms of the number of vertices $(n)$ plus the number of edges $(m)$ of a graph $G$. The upto-date Label $+G$ approaches are GRAIL and Ferrari. However, the Label $+G$ approaches may take long query time when it needs $D F S$ to search the destination vertex over a large graph in $O(n+m)$. On the other hand, there are two main ways taken by the LabelOnly approaches. (1) Some works aim at constructing a small index by compressing TC, because it leads to a small index, and therefore reduces query time $[1,16,13,28,11,12,8,21,20,27]$. (2) Some works aim at constructing an index fast. Different from the Label $+G$ approaches, none of the Label-Only approaches can construct an index in linear time, and the index size by an LabelOnly approach can be either non-linear or be small but cannot be bounded. The up-to-date Label-Only approaches are all from (2) including TF-Label, HL, and DL.

The main contributions of this work are summarized below. First, different from all the existing approaches, as the first, we study a new approach which employs randomness. With the randomness introduced, we can construct an index fast, and answer reachability queries efficiently. Second, we propose a novel labeling, denoted as IP, based on independent permutation [3]. We discuss the ideas, give the algorithms, and show the bounds. Third, we propose two additional labels which can be computed in linear time and used to reduce the search cost when DFS is needed at run-time. Finally, we conduct extensive experimental studies to compare with the up-to-date approaches using 19 large real datasets used in the existing work and synthetic datasets. We confirm the efficiency of our approach.

The remainder of the paper is organized as follows. We discuss the preliminaries and the problem in Section 2, and discuss the related work in Section 3. We give the main ideas of IP labels in Section 4 followed by the discussion on the algorithms to compute IP labels in Section 5. We also discuss two additional labels to 
be used to together with $I P$ at run-time in Section 6. We give our algorithm to answer reachability queries in Section 7 . We report our experimental studies using large real and synthetic datasets in Section 8, and conclude our paper in Section 9.

\section{PRELIMINARIES}

We model a directed graph as $G=(V, E)$ where $V(G)$ represents a set of vertices and $E(G)$ represents a set of edges (ordered pairs of vertices) of $G$. We may simply use $V$ and $E$ if the context is obvious. The numbers of vertices and edges in $G$ are denoted as $n=|V|$ and $m=|E|$, respectively. A path from a vertex $u$ to a vertex $v$ is defined as $\operatorname{path}(u, v)=\left(v_{1}, v_{2}, \cdots, v_{p}\right)$ where $\left(v_{i}, v_{i+1}\right)$ is an edge in $E$, for $1 \leq i<p, u=v_{1}$, and $v=v_{p}$. The length of path $(u, v)$ is the number of edges in the path. The distance between two vertices, $u$ and $v$, denoted as $\operatorname{dst}(u, v)$, is the shortest path distance (length) from $u$ to $v$ in $G$. A vertex $u$ is said to reach $v$ if there exists a path from $u$ to $v$ over $G$. In the following, we use $\operatorname{Out}(u)$ to denote the entire set of vertices that $u$ can reach including $u$ itself, and use $\operatorname{In}(u)$ to denote the entire set of vertices in which every vertex can reach $u$ including $u$ itself. In addition, we use $N_{O}(u)$ and $N_{I}(u)$ to denote the out-neighbors and in-neighbors of $u$, such as $N_{O}(u)=\{v \mid(u, v) \in G\}$ and $N_{I}(u)=\{v \mid(v, u) \in G\}$, respectively. The in-degree, out-degree, and the degree of a vertex $u$ is denoted as, $d_{I}(u)=\left|N_{I}(u)\right|, d_{O}(u)=\left|N_{O}(u)\right|$, and $d(u)=d_{I}(u)+d_{O}(u)$.

A reachability query, denoted as $\operatorname{Reach}(u, v)$, is to answer if $u$ can reach $v$ over $G$. We use $u \rightsquigarrow v$ to denote $u$ can reach $v$ (reachable), and $u \not \sim v$ otherwise.

In this paper, like the existing works, we assume $G$ is a directed acyclic graph (DAG). This is because any directed graph $G$ can be condensed into a DAG, where a vertex in DAG represents a strongly connected component (SCC) in $G$, and an edge $\left(S_{i}, S_{j}\right)$ in DAG represents that there is at least one edge from a vertex in an SCC that $S_{i}$ represents to a vertex in another SCC that $S_{j}$ represents. Therefore, $\operatorname{Reach}(u, v)$ in $G$ can be answered by $\operatorname{Reach}\left(S_{i}, S_{j}\right)$ in the corresponding DAG, if $u$ and $v$ belong to $S_{i}$ and $S_{j}$, respectively. Note that $u$ and $v$ are reachable, $u \rightsquigarrow v$ and $v \rightsquigarrow u$, if $u$ and $v$ co-exist in the same SCC in $G$.

The problem we study in this paper is to answer $\operatorname{Reach}(u, v)$ online when $G$ is very large. It is commonly known that the online breadth/depth-first search (BFS/DFS) algorithms cannot answer $\operatorname{Reach}(u, v)$ for a large graph $G$ efficiently, because both have the time complexity in $O(m+n)$. On the other hand, although it is possible to answer $\operatorname{Reach}(u, v)$ in $O(1)$ by maintaining the edge transitive closure (TC) of $G$, the space complexity for maintaining TC is $O\left(n^{2}\right)$, which is too large for a very large graph $G$, with the time complexity $O(n m)$ to compute TC.

\section{RELATED WORKS}

In general, the main idea to answer $\operatorname{Reach}(u, v)$ online is to construct an index offline, where every entry in the index keeps a label computed for a vertex $u \in G$, denoted label $(u)$. Reach $(u, v)$ can be answered either by Label-Only approach or Label+ $G$ approach. The former is to answer $\operatorname{Reach}(u, v)$ by label $(u)$ and label $(v)$ only, and the latter is to answer $\operatorname{Reach}(u, v)$ by label $(u)$ and label $(v)$ with the possibility of accessing $G$ if needed. There are three main costs, namely, query time (the time to answer online), construction time (the time to construct an index offline), and the index size (the space needed to maintain the index). Here, as indicated in many works, the query time is in the range between $O(1)$ with TC as an index and $O(n+m)$ without any index, the index size is between

\begin{tabular}{llll}
\hline & Query Time & Index Size & Construction Time \\
\hline BFS/DFS & $O(n+m)$ & $O(1)$ & $O(1)$ \\
TC [25, 27] & $O(1)$ & $O\left(n^{2}\right)$ & $O(n m)$ \\
\hline Chain-Cover [8]O(log $k)$ & $O(k n)$ & $O\left(n^{2}+k n \sqrt{k}\right)$ \\
Tree-Cover [1] & $O(\log n)$ & $O\left(n^{2}\right)$ & $O(n m)$ \\
Dual-Label [28] $O(1)$ & $O\left(n+t^{2}\right)$ & $O\left(n+m+t^{3}\right)$ \\
Path-Tree [21] & $O\left(\log ^{2} k\right)$ & $O(k n)$ & $O(k m)$ or $O(n m)$ \\
2-Hop [13] & $O\left(m^{1 / 2}\right)$ & $O\left(n m^{1 / 2}\right)$ & $O\left(n^{3} \cdot|T C|\right)$ \\
3-Hop [20] & $O(\log n+k)$ & $O(k n)$ & $O\left(k n^{2} \cdot|C o n(G)|\right)$ \\
TF-Label [10] & - & - & $O(T)$ \\
HL [19] & - & - & $O(H)$ \\
DL [19] & - & - & $O(n(n+m) L)$ \\
\hline Tree+SSPI [7] & $O(m-n)$ & $O(n+m)$ & $O(n+m)$ \\
GRIPP [26] & $O(m-n)$ & $O(n+m)$ & $O(n+m)$ \\
GRAIL [29] & $O(k)$ or $O(n+m) O(k n)$ & $O(k(n+m))$ \\
Ferrari [24] & $O(k)$ or $O(n+m) O((k+s) n) O\left(k^{2} m+S\right)$ \\
\hline IP+ (ours) & $O(k)$ or $O\left(k n r^{2}\right)$ & $O((k+h) n) O((k+h)(m+n))$ \\
\hline
\end{tabular}

Table 1: Time and Space Complexity $\left(T=\sum_{1 \leq i \leq \log \ell(G)}\right.$ $\left.\sum_{v \in V\left(G_{i}^{*}\right) \backslash V\left(G_{i+1}\right)} h(v), H=\sum_{1 \leq i<h}\left(\sum_{v \in V\left(B_{i}\right) \backslash V\left(B_{i+1}\right)} g(v)\right)\right)$

$O\left(n^{2}\right)$ with TC and $O(1)$ without an index computed, and the time complexity for index construction can be very high, when it aims at compressing TC to minimal. A survey can be found in [31]. Table 1 summarizes the three main costs for the existing work.

Label-Only: Several approaches proposed aim at finding a way to compress TC, because a smaller index size implies labels computed for vertices are smaller, which leads to a better query time. Jagadish [16] first introduces chain decomposition, which is also known as Chain-Cover, to compress TC by finding a minimal number of pair-wise disjoint chains to represent DAG. Here, a chain is a sequence of $\left(v_{1}, v_{2}, \cdots, v_{c}\right)$ in which $v_{i} \rightsquigarrow v_{j}$ if $i \leq j$. In $G$, every vertex $v$ is assigned to a pair of $\left(c_{i}, p_{j}\right)$ where $c_{i}$ is the chain id $v$ belongs to and $p_{j}$ is the position of $v$ in $c_{i}$, and label $(u)$ is a set of such pairs. By Chain-Cover, $u \rightsquigarrow v$ if there is a pair $\left(c_{i}, p_{j}\right)$ in label $(u)$, such that $v$ is in the chain $c_{i}$ and its position is $\geq p_{j}$. Let $k$ be the minimal number of chains in DAG, the query time of Chain-Cover is $O(\log k)$. Chen and Chen propose algorithms in [8] to compute Chain-Cover to construct an index of $O(k n)$ in $O\left(n^{2}+k n \sqrt{k}\right)$ time. Agrawal et al. propose TreeCover [1] that covers TC using an optimal spanning tree, based on which a vertex, $v$, is assigned to an interval $[s, e]$, where $e$ is the postorder of $v$, and $s$ is the smallest postorder of $v$ 's descendants. Here, label $(u)$ in Tree-Cover is a set of intervals, and $u \rightsquigarrow v$ if the interval of $v$ is fully contained in an interval in label $(u)$. The query time is $O(\log n)$, but its index size is $O\left(n^{2}\right)$ and the construction time is $O(n m)$, which are both high. Wang et al. propose Dual-Label [28] for a sparse graph. By Dual-Label, label $(v)$ has two parts. One is a single interval for answering reachability over a spanning tree of $G$, and the other is to deal with the transitive closure over the non-tree edges of $G$. Let the number of non-tree edges be $t$. The transitive closure is $O\left(t^{2}\right)$ for $t \ll n$ over a sparse graph $G$. Dual-Label can achieve $O(1)$ query time, since it works as to maintain TC. Dual-Label is constructed in $O\left(n+t^{2}\right)$ time and in $O\left(n+m+t^{3}\right)$ space. When $G$ becomes denser, $t$ will approach $n$. Jin et al. propose Path-Tree [18], which decomposes a DAG $G$ into a set of pair-wise disjoint paths. Path-Tree shares the similar ideas used in Chain-Cover. But, unlike Chain-Cover, Path-Tree uses paths instead of chains. The query time is $O\left(\log ^{2} k\right)$ where $k$ is the number of paths computed in Path-Tree. Path-Tree can have a smaller index size than Chain-Cover. van Schaik and de Moor propose a bit-vector approach to compress TC [27]. Even though many approaches make use of a spanning tree to construct an index using intervals (a pair of numbers), the construction time is non-linear and cannot deal with larger and denser graphs. 
Cohen et al. in [13] propose 2-Hop label. A label $(u)$ consists of $L_{\text {out }}(u)$ and $L_{\text {in }}(u)$, where $L_{\text {out }}(u)$ is a subset of vertices that $u$ can reach $\left(L_{\text {out }}(u) \subseteq O u t(u)\right)$ and $L_{\text {in }}(u)$ is a subset of those vertices that can reach $u\left(L_{i n}(u) \subseteq \operatorname{In}(u)\right)$. 2-Hop compresses TC. By 2-Hop, $u \rightsquigarrow v$ if and only if $L_{\text {out }}(u) \cap L_{\text {in }}(v) \neq \emptyset$. Computing the optimal 2-Hop for $G$ is known to be NP-hard [13], since it is a set-cover problem. In [13], an approximate (greedy) algorithm is proposed. Several heuristic approaches are proposed to compute 2-Hop [23, 11, 12]. Furthermore, Jin et al. propose 3-Hop [20] to improve 2-Hop by utilizing the idea of the chain decomposition as used in Chain-Cover. It takes $O(\log n+k)$ query time, but it takes $O\left(k n^{2} \cdot|C o n(G)|\right)$ time to construct 3-Hop with the space complexity of $O(k n)$, where $k$ is the number of chains and $\operatorname{Con}(G)$ is the transitive closure contour (Definition 3 in [20]). Cai et al. propose Path-Hop [5] to replace the chain decomposition with a tree structure to improve 3-Hop.

Cheng et al. [10] propose TF-Label (topological folding) to compute 2-Hop labels for a DAG $G$ using topological level. A vertex in $G$ has a level assigned, denoted as $\ell(u, G)$, where the min level is 1 , and the max level is $\ell(G)$. Given the levels, $V(G)$ can be represented as a disjoint set of vertices, such that $V(G)=\cup_{i=1}^{\ell(G)} L_{i}(G)$, where $L_{i}(G)$ is the set of vertices at the level $i$. Furthermore, $G$ can be represented as a sequence of DAGs, such that $\mathbb{G}=\left(G_{1}, G_{2}\right.$, $\left.\cdots G_{f}\right)$, for $f=\left\lfloor\log _{2} \ell(G)\right\rfloor+1$. Here, $G_{1}=G$, and $G_{i}$ for $i>1$ is constructed as follows: $V\left(G_{i}\right)=\cup_{1 \leq j \leq\left\lfloor\ell\left(G_{i-1}\right) / 2\right\rfloor} L_{2 j}\left(G_{i-1}\right)$ and $E\left(G_{i}\right)$ is a set of edges, $(u, v)$, over $V\left(G_{i}\right)$ if $u \rightsquigarrow v$ in the original $G$ and $\ell\left(v, G_{i}\right)=\ell\left(u, G_{i}\right)+1$ (non-cross-edge). For example, suppose a DAG $G$ has 6 levels $\ell(G)=6$. Then, $G_{1}=G . G_{2}$ is a DAG with 3 levels consisting of all vertices at the level 2, 4, and 6 of $G_{1}$, and all non-cross-edges. $G_{3}$ is a DAG with 1 level. Since $G_{i}$ may have cross-edges from a vertex at the level $i$ to a vertex at level $j$ for $j>i+1$, TF-Label transforms $G_{i}$ to $G_{i}^{*}$ such all edges in $G_{i}^{*}$ are from a vertex at the level $i$ to a vertex at the level $i+1$ by adding dummy vertices. The topological folding reduces the computational cost to compute 2-Hop labels. The bound for index construction time given [10] is $O\left(\sum_{1 \leq i \leq \log \ell(G)} \sum_{v \in V\left(G_{i}^{*}\right) \backslash V\left(G_{i+1}\right)} h(v)\right)$, where $h(\cdot)$ is the cost of computing 2-Hop label for $v$. It is worth noting that the time complexity of TF-Label is non-linear, because it needs to merge 2Hop labels for a vertex $v$ in $G_{i+1}$ to $G_{i}$. Also because TF-Label aims at computing 2-Hop labels efficiently instead of attempting to minimizing the index size, there is no tight bound for the index size, and the time complexity for query time depends on the index size.

Jin et al. [17] propose $S C A R A B$ as a general framework to represent a reachability backbone, denoted as a graph $B(V, E)$, for a graph $G$. The reachability backbone $B$ represents every $u \rightsquigarrow v$ that are reachable in $G$, if $u$ and $v$ exist in $B$, given $V(B) \subseteq V(G)$. With the reachability backbone $B, \operatorname{Reach}(u, v)$ for $u$ and $v$ in $G$ can be answered. That is, $u \rightsquigarrow v$, if there exists a pair of local neighbor vertices, $u^{\prime}$ and $v^{\prime}$, such that $\operatorname{dst}\left(u, u^{\prime}\right) \leq \epsilon, u^{\prime} \rightsquigarrow v^{\prime}$ in $B$, and $\operatorname{dst}\left(v^{\prime}, v\right) \leq \epsilon$. Any algorithms can be used to construct an index over $B$ which is smaller than $G$. In [19], Jin et al. further propose $H L$ (Hierarchical Labeling) over SCARAB. Informally, a graph $G$ can be represented as a sequence of reachability backbones, $\mathbb{B}=\left(B_{0}, B_{1}, B_{2}, \cdots, B_{h}\right)$, where $B_{0}=G$ and $B_{i+1}$ is the reachability backbone of $B_{i}$, for $i>0$. With HL, the 2-Hop labels computed for $B_{i+1}$ can be merged to $B_{i}$. The time complexity of $H L$ construction time is $O\left(\sum_{1 \leq i<h}\left(\sum_{v \in V\left(B_{i}\right) \backslash V\left(B_{i+1}\right)} g(v)\right)\right.$, where $h$ is the number of backbones, and $g(\cdot)$ is the cost of computing 2-Hop label for $v$. Also, in [19], Jin et al. propose $D L$ (Distribution Labeling). It is based on a list of vertices in an order
$\mathbf{V}=\left(v_{1}, v_{2}, \cdots, v_{n}\right)$. Given $\mathbf{V}$, the 2-Hop labels are computed by breadth-first search (BFS) for every vertex $v_{j}$ twice: BFSforward following the direction of edges and BFS-backward following the reversed direction of edges, with an early stop condition. First, we assign $v_{1}$ for every vertex in $\operatorname{Out}\left(v_{1}\right)$ and $\operatorname{In}\left(v_{1}\right)$. That is, $L_{\text {in }}(w)=\left\{v_{1}\right\}$ for every $w \in O u t\left(v_{1}\right)$, and $L_{\text {out }}(u)=\left\{v_{1}\right\}$ for every $u \in \operatorname{In}\left(v_{1}\right)$. Second, consider BFS of $v_{i}$ in $\mathbf{V}$ for $i>1$. $v_{i}$ will be inserted into $L_{i n}\left(v_{i}\right)$. Assume the BFS-forward reaches $v_{j}$ from $v_{i}, v_{i}$ will be inserted into $L_{i n}\left(v_{j}\right)$ if $L_{i n}\left(v_{j}\right) \cap L_{\text {out }}\left(v_{i}\right)=\emptyset$, otherwise $B F S$-forward will not continue from $v_{j}$. In a similar way, assume the BFS-backward reaches $v_{j}$ from $v_{i}, v_{i}$ will be inserted into $L_{\text {out }}\left(v_{j}\right)$ if $L_{\text {out }}\left(v_{j}\right) \cap L_{\text {in }}\left(v_{i}\right)=\emptyset$, otherwise BFSbackward will not continue from $v_{j}$. The early stop condition makes the 2-Hop labels for $G$ compact. The time complexity of $D L$ is $O(n(n+m) L)$ where $L$ is the maximal labeling size. However, like TF-Label, HL and DL aim at reducing the construction time to compute 2-Hop labels, and the index size and the query time are not tightly bounded.

Label+G: Tree+SSPI [7] and GRIPP [26] are two works that use an interval label for every vertex over a spanning tree, and attempt to reduce depth-first-search (DFS) time if needed at run-time. The query time for Tree+SSPI and GRIPP are $O(m-n)$, and both construction time and index size are $O(n+m)$.

Yildirim et al. propose GRAIL [29, 30], which randomly generates $k$ DFS spanning trees to cover $G$, which can significantly reduce query time than that of using a single spanning tree. The label of a vertex is $k$ intervals, label $(v)=\left(I_{1}, I_{2}, \cdots, I_{k}\right)$, where the $j$-th interval $I_{j}=\left[s_{j}, e_{j}\right]$ is computed by the $j$-th DFS. Let label $(v) \subseteq$ label $(u)$ if all intervals $I_{j}$ of $v$ are contained in $I_{j}$ of $u$ computed in the same DFS, and label $(v) \nsubseteq$ label $(u)$ otherwise. GRAIL can only answer $u \not \psi v$ over $G$ if label $(v) \nsubseteq$ label $(u)$. But, GRAIL needs to do DFS from $u$ to reach $v$ at run-time, if label $(v) \subseteq$ label $(u)$. The DFS from $u$ does not need to continue at a vertex $w$, if label $(v) \nsubseteq$ label $(w)$. The query time is either $O(k)$ using the label only or $O(n+m)$ when it needs to do DFS.

Seufert et al. propose Ferrari [24]. Like GRAIL, Ferrari computes up to $k$ intervals for every vertex over an optimal spanning tree computed by [1]. Let label $(u)=\left(I_{1}, I_{2}, \cdots\right)$ be the set of intervals for $u$. It is worth noting that by Tree-Cover the number of intervals for a vertex cannot be bounded, but in order to control the index size Ferrari only assigns up to $k$ intervals for a vertex. Therefore, some intervals in label $(u)$ are approximate intervals, because they cover certain interval that is not supposed to be covered to correctly answer Reach $(u, v)$. Ferrari is to minimize the size of approximate intervals. This is done by computing up to $k$ intervals of $u$ with the intervals computed for all children of $u$ using dynamic programming. The Ferrari constructed is not optimal. The time complexity for construction is $O\left(k^{2} m+S\right)$, where $S$ is the time complexity of finding the top- $s$ largest degree vertex for seed based pruning and constructing the index. Its index size is $O((k+s) n)$, where $s$ is the number of seeds added to every vertex label.

\section{A NEW LINEAR LABELING}

As discussed in Section 3, in the category of the Label-Only approaches, the approaches that aim at compressing TC, like 2-Hop and 3-Hop, incur high construction cost and large index size, which affects query time. Chain-Cover and Tree-Cover use a disjoint set of chains and a tree cover, respectively, but they cannot deal with large dense graphs. Dual-Label can reach $O(1)$ query time in theory, but it is for a considerably sparse graph. The up-to-date approaches in this category are TF-Label, HL, and DL. They aim at reducing the construction time, but the construction time is non- 
linear, which will incur high construction time for large and dense graphs. In addition, both index size and query time cannot be tightly bounded.

In comparison with the Label-Only approaches, in the category of Label $+G$, all approaches are linear regarding construction time and index size. Here, linear is on the basis of the graph size (the number of vertices plus the number of edges). Given the linear construction time and index size, the query time for Tree+SSPI and GRIPP is $O(m-n)$ and the query time for GRAIL and Ferrari can be up to $O(n+m)$, which means it cannot deal with large dense graphs. In $[10,19]$, the authors indicate that TF-Label, HL, and DL outperform GRAIL, in terms of query time in practice.

The main idea: In this paper, we propose a novel labeling approach, called IP (Independent Permutation), and test set containment with probability guarantee, where the label of a vertex is a set. Let $\mathcal{A}$ and $\mathcal{B}$ be two subsets of a set $\mathcal{V}$. There are two ways to check whether $\mathcal{B}$ is contained in $\mathcal{A}$. One is to check $\mathcal{B} \subseteq \mathcal{A}$, and the other is to check $\mathcal{B} \nsubseteq \mathcal{A}$. Both are time consuming if an exact answer is needed for large sets. We take the latter approach, and answer a reachability query by finding whether there is at least one element in one set that is not contained in the other for $\mathcal{B} \nsubseteq \mathcal{A}$. Our approach is based on randomness with high probability guarantee where the guarantee is ensured by independent permutation. With the probability guarantee, we can answer a large number of $\mathcal{B} \nsubseteq \mathcal{A}$ tests with $100 \%$ probability guarantee by IP. For those we cannot answer by $I P$ with $100 \%$ probability guarantee, we do DFS online. It is important to note that the randomness we use is to minimize the probability of doing DFS, and the randomness is based on min-wise independent permutations [4]. We further improve the probability guarantee by min-wise independent permutations with a new $k$-min-wise independent permutations proposed in this work.

Before introducing min-wise independent permutations followed by our $k$-min-wise independent permutations, we show how $u \rightsquigarrow v$ is connected to $\mathcal{A}$ and $\mathcal{B}$ in IP. This is based on our observation that $u \rightsquigarrow v$, if $\operatorname{Out}(v) \subseteq \operatorname{Out}(u)$ or $\operatorname{In}(u) \subseteq \operatorname{In}(v)$. In other words, if $u$ can reach all the vertices that $v$ can reach, or all vertices that can reach $u$ can also reach $v$, then $u \rightsquigarrow v$. In a similar manner, $u \not \leftrightarrow v$, if $\operatorname{Out}(v) \nsubseteq \operatorname{Out}(u)$ or $\operatorname{In}(u) \nsubseteq \operatorname{In}(v)$. Therefore, $\mathcal{A}$ and $\mathcal{B}$ denote $\operatorname{Out}(u)$ and $\operatorname{Out}(v)$ respectively, or $\operatorname{In}(v)$ and $\operatorname{In}(u)$ respectively. Below, we consider $\mathcal{A}$ and $\mathcal{B}$ as a subset of $\mathcal{V}$ which is a set of $n$ numbers, $\mathcal{V}=\{0,1,2, \cdots, n-1\}$, representing all vertices in $V(G)$ for $n=|V(G)|$.

Min-wise Independent Permutations: We briefly review the minwise independent permutations as given [4]. Given a set of numbers, $\Omega$, a permutation of $\Omega$ is a bijection from $\Omega$ to itself, denoted as $\pi: \Omega \rightarrow \Omega$. It is important to note that $\pi(x)$, for $x \in \Omega$, is unique in $\pi(\Omega)$. Consider $\mathcal{V}$ as a set of numbers of size $n$. Let $\mathcal{V}_{n}$ be the set of all permutations of $\mathcal{V} . \mathcal{F} \subseteq \mathcal{V}_{n}$ is min-wise independent, if for any $X \subseteq \mathcal{V}$ and any $x \in X$, when $\pi$ is given to choose uniformly and randomly from $\mathcal{F}$, then the following holds:

$$
\operatorname{Pr}(\min \{\pi(X)\}=\pi(x))=\frac{1}{|X|}
$$

This says that, for any $x \in X$, the probability that $\pi(x)$ is the smallest number in $\pi(X)$ is $1 /|X|$. Based on the min-wise independent permutations, Min-Hash [3] is designed to compute Jaccard similarity of two sets, $\mathcal{A}$ and $\mathcal{B}, \operatorname{Jacard}(\mathcal{A}, \mathcal{B})=|\mathcal{A} \cap \mathcal{B}| /|\mathcal{A} \cup \mathcal{B}|$ as $\operatorname{Pr}(\min \{\pi(\mathcal{A})\}=\min \{\pi(\mathcal{B})\})$

In this work, our focus is on set containment, $\mathcal{B} \subseteq \mathcal{A}$. We know that $\mathcal{B} \subseteq \mathcal{A}$ if $\mathcal{A}=\mathcal{A} \cup \mathcal{B}$. Let $\min \{X\}$ indicates the smallest number of a set of numbers, $X$, we have

$$
\operatorname{Pr}(\min \{\pi(\mathcal{A} \cup \mathcal{B})\}=\min \{\pi(\mathcal{B} \backslash \mathcal{A})\})=\frac{|\mathcal{B} \backslash \mathcal{A}|}{|\mathcal{A} \cup \mathcal{B}|}=1-\frac{|\mathcal{A}|}{|\mathcal{A} \cup \mathcal{B}|}
$$

By min-wise independent permutations, any number in $\mathcal{A} \cup \mathcal{B}$ is equally likely to have the smallest number in $\pi(\mathcal{A} \cup \mathcal{B})$, e.g., $\min \{$ $\pi(\mathcal{A} \cup \mathcal{B})\}$, and $\min \{\pi(\mathcal{A} \cup \mathcal{B}\})=\min \{\pi(\mathcal{B} \backslash \mathcal{A})\}$ is true, if and only if $\min \{\pi(\mathcal{A})\}>\min \{\pi(\mathcal{B})\}$. Hence, we have

$$
\operatorname{Pr}(\min \{\pi(\mathcal{A})\}>\min \{\pi(\mathcal{B})\})=1-\frac{|\mathcal{A}|}{|\mathcal{A} \cup \mathcal{B}|}
$$

Because $\mathcal{B} \subseteq \mathcal{A}$ if $|\mathcal{A}|=|\mathcal{A} \cup \mathcal{B}|$, there does not exist any possible permutation $\pi$ by which $\min \{\pi(\mathcal{A})\}>\min \{\pi(\mathcal{B})\}$, as given in Eq. (3). Therefore, the condition of $\min \{\pi(\mathcal{A})\}>\min \{\pi(\mathcal{B})\}$ can be used to conclude $\mathcal{B} \nsubseteq \mathcal{A}$. However, there are cases that $\mathcal{B} \nsubseteq \mathcal{A}$ is true, when $\min \{\pi(\mathcal{A})\}>\min \{\pi(\mathcal{B})\}$ is not true. As can be seen from Eq. (3), the probability for $\min \{\pi(\mathcal{A})\}>$ $\min \{\pi(\mathcal{B})\}$ to be false is non-zero, $\frac{|\mathcal{A}|}{|\mathcal{A} \cup \mathcal{B}|}$. In other words, when $\min \{\pi(\mathcal{A})\}>\min \{\pi(\mathcal{B})\}$ is not true, it can be possible that either $\mathcal{B} \subseteq \mathcal{A}$ or $\mathcal{B} \nsubseteq \mathcal{A}$. Here, our problem to answer $u \not \iota v$ by $\mathcal{B} \nsubseteq \mathcal{A}$ becomes a problem on how to increase the probability for $\min \{\pi(\mathcal{A})\}>\min \{\pi(\mathcal{B})\}$ to be true.

$k$-min-wise: In order to increase $\operatorname{Pr}(\min \{\pi(\mathcal{A})\}>\min \{\pi(\mathcal{B})\})$, for testing $\mathcal{B} \subseteq \mathcal{A}$, we propose to use top- $k$ smallest number$\mathrm{s}$ instead of the top-1 smallest number as used in min-wise independent permutation. By independent permutation $\pi$, we define $\min _{k}\{\pi(X)\}$ as a subset of $\pi(X)$ containing up to $k$ smallest numbers, such as $\min _{k}\{\pi(X)\}=\left(\pi\left(x_{1}\right), \pi\left(x_{2}\right), \cdots\right)$, where $\pi\left(x_{i}\right)<\pi\left(x_{j}\right)$ if $i<j$, and $\left|\min _{k}\{\pi(X)\}\right| \leq k$, Here, by $k$-min-wise $\left(\min _{k}\right)$, we mean $\pi\left(x_{i}\right)<\pi\left(x_{j}\right)$ for any $\pi\left(x_{i}\right) \in$ $\min _{k}\{\pi(X)\}$ and any $\pi\left(x_{j}\right) \in \pi(X) \backslash \min _{k}\{\pi(X)\}$.

To deal with $\mathcal{B} \subseteq \mathcal{A}$, we define an order $(\preceq)$ between $\min _{k}\{\pi(\mathcal{A})\}$ and $\min _{k}\{\pi(\mathcal{B})\}$, such that $\min _{k}\{\pi(\mathcal{A})\} \preceq \min _{k}\{\pi(\mathcal{B})\}$ if every $\pi\left(b_{i}\right) \in \min _{k}\{\pi(\mathcal{B})\} \backslash \min _{k}\{\pi(\mathcal{A})\}$ is greater than the largest number in $\min _{k}\{\pi(\mathcal{A})\}$. We use $\min _{k}\{\pi(\mathcal{A})\} \succ \min _{k}\{\pi(\mathcal{B})\}$ otherwise. We prove $\mathcal{B} \nsubseteq \mathcal{A}$, if $\min _{k}\{\pi(\mathcal{A})\} \succ \min _{k}\{\pi(\mathcal{B})\}$ in Theorem 4.1.

Theorem 4.1: Let $\mathcal{A}$ and $\mathcal{B}$ be a subset of $\mathcal{V}=\{0,1,2, \cdots, n-$ $1\}$, and $\pi$ be randomly and uniformly chosen from $\mathcal{V}_{n}$ (the set of all permutations of $\mathcal{V}$ ). Then, $\mathcal{B} \nsubseteq \mathcal{A}$, if $\min _{k}\{\pi(\mathcal{A})\} \succ$ $\min _{k}\{\pi(\mathcal{B})\}$.

Proof Sketch: Consider the case when $\mathcal{B} \subseteq \mathcal{A}$. We have $\pi(\mathcal{B}) \subseteq$ $\pi(\mathcal{A})$. Because the $k$ smallest numbers in $\min _{k}\{\pi(\mathcal{A})\}$ are taken from $\pi(\mathcal{A})$ or in other words taken from $\pi(\mathcal{A} \cup \mathcal{B})$, there cannot exist $\pi\left(b_{i}\right)$ in $\min _{k}\{\pi(\mathcal{B})\} \backslash \min _{k}\{\pi(\mathcal{A})\}$ that is smaller than the largest number in $\min _{k}\{\pi(\mathcal{A})\}$. Therefore, if $\mathcal{B} \subseteq \mathcal{A}$, we have $\min _{k}\{\pi(\mathcal{A})\} \preceq \min _{k}\{\pi(\mathcal{B})\}$. Hence, if $\min _{k}\{\bar{\pi}(\mathcal{A})\} \succ$ $\min _{k}\{\pi(\mathcal{B})\}$, then $\mathcal{B} \not \mathcal{A}$.

Next, like Eq. (3), we give the probability for $\min _{k}\{\pi(\mathcal{A})\} \succ$ $\min _{k}\{\pi(\mathcal{B})\}$ in Theorem 4.2.

Theorem 4.2: Let $\mathcal{A}$ and $\mathcal{B}$ be a subset of $\mathcal{V}=\{0,1,2, \cdots, n-$ $1\}$, and $\pi$ be randomly and uniformly chosen from $\mathcal{V}_{n}$ (the set of all permutations of $\mathcal{V})$. Assume $|\mathcal{A}|=p,|\mathcal{A} \cup \mathcal{B}|=q$, and $\left|\min _{k}\{\pi(\mathcal{A})\}\right|=k_{A}$ for $k_{A} \leq k$.

$$
\operatorname{Pr}\left(\min _{k}\{\pi(\mathcal{A})\} \succ \min _{k}\{\pi(\mathcal{B})\}\right)=1-\frac{p !\left(q-k_{A}\right) !}{q !\left(p-k_{A}\right) !}
$$

Proof Sketch: For $\mathcal{B} \subseteq \mathcal{A}, \operatorname{Pr}\left(\min _{k}\{\pi(\mathcal{A})\} \succ \min _{k}\{\pi(\mathcal{B})\}\right)=$ 0 because $p=q$. We focus on the cases for $\mathcal{B} \nsubseteq \mathcal{A}$, provided $\mathcal{X}=\mathcal{A} \cup \mathcal{B}$.

Recall that for a given $k$, "up to $k$ " is for the cases that the size of a set can be less than $k$. In general, $|\mathcal{A}|,|\mathcal{B}|$, and $|\mathcal{X}|$ can be possible $\leq k$. We use $k_{A}, k_{B}$, and $k_{X}$, which are a max possible number $\leq k$, for $\left|\min _{k}\{\pi(\mathcal{A})\}\right|=k_{A},\left|\min _{k}\{\pi(\mathcal{B})\}\right|=k_{B}$, and $\left|\min _{k}\{\bar{\pi}(\mathcal{X})\}\right|=k_{X}$, because $k_{A} \leq|\mathcal{A}|, k_{B} \leq|\mathcal{B}|$, and $k_{X} \leq$ $|\mathcal{X}|$. We also know $k_{A} \leq k_{X}$ and $k_{B} \leq k_{X}$ because $\mathcal{X}$ is the union 


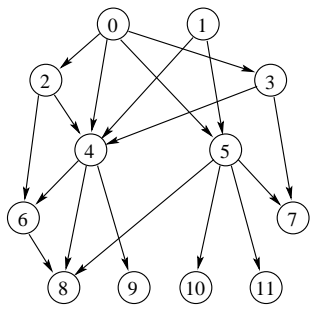

(a) DAG $G$

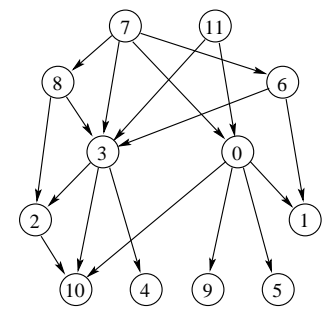

(b) A permutation $\pi$
Figure 1: A permutation $\pi$

\begin{tabular}{|c|l|l||c|l|l|}
\hline Vertex & $\mathcal{L}_{\text {out }}$ & $\mathcal{L}_{\text {in }}$ & Vertex & $\mathcal{L}_{\text {out }}$ & $\mathcal{L}_{\text {in }}$ \\
\hline \hline$v_{0}$ & $\{0,1,2,3,4\}$ & $\{7\}$ & $v_{6}$ & $\{2,10\}$ & $\{2,3,6,7,8\}$ \\
\hline$v_{1}$ & $\{0,1,2,3,4\}$ & $\{11\}$ & $v_{7}$ & $\{1\}$ & $\{0,1,6,7,11\}$ \\
\hline$v_{2}$ & $\{2,3,4,8,10\}$ & $\{7,8\}$ & $v_{8}$ & $\{10\}$ & $\{0,2,3,6,7\}$ \\
\hline$v_{3}$ & $\{1,2,3,4,6\}$ & $\{6,7\}$ & $v_{9}$ & $\{4\}$ & $\{3,4,6,7,8\}$ \\
\hline$v_{4}$ & $\{2,3,4,10\}$ & $\{3,6,7,8,11\}$ & $v_{10}$ & $\{9\}$ & $\{0,7,9,11\}$ \\
\hline$v_{5}$ & $\{0,1,5,9,10\}$ & $\{0,7,11\}$ & $v_{11}$ & $\{5\}$ & $\{0,5,7,11\}$ \\
\hline
\end{tabular}

Table 2: IP Label for DAG $G$ given in Fig. 1

of $\mathcal{A}$ and $\mathcal{B}$. For simplicity, we use $\min _{k_{A}}\{\pi(\mathcal{A})\}, \min _{k_{B}}\{\pi(\mathcal{B})\}$, and $\min _{k_{X}}\{\pi(\mathcal{X})\}$, to indicates $\min _{k}\{\pi(\mathcal{A})\}, \min _{k}\{\pi(\mathcal{B})\}$, and $\min _{k}\{\pi(\mathcal{X})\}$, whose sizes are $k_{A}, k_{B}$, and $k_{X}$.

By Theorem 4.1, we know that if all the smallest numbers in $\min _{k_{A}}\{\pi(\mathcal{A})\}$ are the top- $k_{A}$ smallest numbers in $\min _{k_{X}}\{\pi(\mathcal{X})\}$, such that $\min _{k_{A}}\{\pi(\mathcal{A})\}=\min _{k_{A}}\{\pi(\mathcal{X})\}$, then $\min _{k}\{\pi(\mathcal{A})\} \preceq$ $\min _{k}\{\pi(\mathcal{B})\}$. In a similar way, it is also true $\min _{k}\{\pi(\mathcal{A})\}=$ $\min _{k_{A}}\{\pi(\mathcal{X})\}$ if $\min _{k}\{\pi(\mathcal{A})\} \preceq \min _{k}\{\pi(\mathcal{B})\}$.

Recall that $\pi$ is equally likely to be any permutation in the set of all permutations of $\mathcal{X}$, denoted $\mathcal{X}_{n}$. We consider the probability of $\min _{k_{A}}\{\pi(\mathcal{A})\}=\min _{k_{A}}\{\pi(\mathcal{X})\}$. There are $\frac{q !}{\left(q-k_{A}\right) !}$ permutations in total to have $\min _{k_{A}}\{\pi(\mathcal{X})\}$. Among such $\frac{q !}{\left(q-k_{A}\right) !}$ permutations, there are $\frac{p !}{\left(p-k_{A}\right) !}$ for $\min _{k_{A}}\{\pi(\mathcal{A})\}=\min _{k_{A}}\{\pi(\mathcal{X})\}$ in total. As a result, the probability that $\min _{k}\{\pi(\mathcal{A})\} \succ \min _{k}\{\pi(\mathcal{B})\}$ is $1-\frac{p !\left(q-k_{A}\right) !}{q !\left(p-k_{A}\right) !}$.

In practice, $k_{A}$ is up to $k$ which is small and is user-controlled. $p(=|\mathcal{A}|)$ and $q(=|\mathcal{A} \cup \mathcal{B}|)$ can be large considering a large graph $G$. We have $k_{A} \ll p \leq q$. When it is the case, the probability that $\min _{k}\{\pi(\mathcal{A})\} \succ \min _{k}\{\pi(\mathcal{B})\}$ can be represented as

$$
1-\frac{p(p-1) \cdots\left(p-k_{A}+1\right)}{q(q-1) \cdots\left(q-k_{A}+1\right)} \approx 1-\left(\frac{p}{q}\right)^{k_{A}}
$$

Here, $\left(\frac{p}{q}\right)^{k_{A}}$ can be very small, even for a relatively small $k_{A}$. Comparing Eq. (4) with Eq. (3), $\operatorname{Pr}\left(\min _{k}\{\pi(\mathcal{A})\} \succ \min _{k}\{\pi(\mathcal{B})\}\right)$ is much larger than $\operatorname{Pr}(\min \{\pi(\mathcal{A})\}>\min \{\pi(\mathcal{B})\})$, and is equal to $\operatorname{Pr}(\min \{\pi(\mathcal{A})\}>\min \{\pi(\mathcal{B})\})$ when $k_{A}=1$.

The IP Labeling: By $k$-min-wise independent permutations, for a vertex $u \in G$, for a given $k$, we define label $(u)$ as a pair of sets, $\mathcal{L}_{\text {out }}(u)$ and $\mathcal{L}_{\text {in }}(u)$. $\mathcal{L}_{\text {out }}(u)$ keeps up to $k$ smallest numbers by the permutation $\pi$ for the set of $\operatorname{Out}(u)$, denoted as $\mathcal{L}_{\text {out }}(u)=$ $\min _{k}\{\pi($ Out $(u))\} . \mathcal{L}_{\text {in }}(u)$ keeps up to $k$ smallest number by the same permutation $\pi$ for the set of $\operatorname{In}(u)$, denoted as $\mathcal{L}_{i n}(u)=$ $\min _{k}\{\pi(\operatorname{In}(u))\}$. By Theorem 4.1, to answer Reach $(u, v), u \not \psi$ $v$ if $\mathcal{L}_{\text {out }}(u) \succ \mathcal{L}_{\text {out }}(v)$ or $\mathcal{L}_{\text {in }}(v) \succ \mathcal{L}_{\text {in }}(u)$ by the labels only. However, it needs to check by DFS online otherwise whether $u \rightsquigarrow$ $v$ or $u \ngtr \leftrightarrow v$ since the probability given in Eq. (4) is non-zero.

Example 4.1: A DAG $G$ is shown in Fig. 1(a). The numbers in Fig. 1(a) identify the 12 vertices, $V(G)=\left\{v_{0}, v_{1}, \cdots, v_{11}\right\}$. With a permutation $\pi$, which we will show how to compute, we assume the result is $\pi(0)=7, \pi(1)=11, \pi(2)=8, \pi(3)=6, \pi(4)=3$, $\pi(5)=0, \pi(6)=2, \pi(7)=1, \pi(8)=10, \pi(9)=4, \pi(10)=$ 9 , and $\pi(11)=5$. Fig. 1(b) shows the $\pi\left(v_{i}\right)$ for every $v_{i} \in$ $V(G)$. Let $k=5$. Table 2 shows $I P$ labels of $G$ given in Fig. 1 . We show four reachability queries. $\left(Q_{1}\right)$ consider $\operatorname{Reach}\left(v_{2}, v_{7}\right)$. We have $\mathcal{L}_{\text {out }}\left(v_{2}\right)=\{2,3,4,8,10\}$ and $\mathcal{L}_{\text {out }}\left(v_{7}\right)=\{1\}$. Since $1 \in \mathcal{L}_{\text {out }}\left(v_{7}\right), 1 \notin \mathcal{L}_{\text {out }}\left(v_{2}\right)$, and $1<$ the largest number 10 in $\mathcal{L}_{\text {out }}\left(v_{2}\right)$, we have $\mathcal{L}_{\text {out }}\left(v_{2}\right) \succ \mathcal{L}_{\text {out }}\left(v_{7}\right)$. By Theorem 4.1, we have $\operatorname{Out}\left(v_{7}\right) \nsubseteq \operatorname{Out}\left(v_{2}\right)$, and therefore $v_{2} \not \iota^{\prime} v_{7}$. $\left(Q_{2}\right)$ consider $\operatorname{Reach}\left(v_{3}, v_{2}\right)$. We have $\mathcal{L}_{\text {out }}\left(v_{3}\right)=\{1,2,3,4,6\}$, $\mathcal{L}_{\text {out }}\left(v_{2}\right)=\{2,3,4,8,10\}$. By definition, $\mathcal{L}_{\text {out }}\left(v_{3}\right) \preceq \mathcal{L}_{\text {out }}\left(v_{2}\right)$. However, since $\mathcal{L}_{i n}\left(v_{2}\right)=\{7,8\}$ and $\mathcal{L}_{i n}\left(v_{3}\right)=\{6,7\}$, we have $\mathcal{L}_{i n}\left(v_{2}\right) \succ \mathcal{L}_{i n}\left(v_{3}\right)$. Because $\operatorname{In}\left(v_{3}\right) \nsubseteq \operatorname{In}\left(v_{2}\right)$, the answer of $\operatorname{Reach}\left(v_{3}, v_{2}\right)$ is $v_{3} \not \nrightarrow v_{2} .\left(Q_{3}\right)$ consider $\operatorname{Reach}\left(v_{1}, v_{8}\right)$. We have $\mathcal{L}_{\text {out }}\left(v_{1}\right) \preceq \mathcal{L}_{\text {out }}\left(v_{8}\right)$ and $\mathcal{L}_{\text {in }}\left(v_{8}\right) \preceq \mathcal{L}_{\text {in }}\left(v_{1}\right)$. We cannot answer it by IP labels only, and DFS is needed over $G$ (Fig. 1(a)). We start DFS from $v_{1}$. Supposed the next vertex to be visited is $v_{4}$. We have to further DFS, because $\mathcal{L}_{\text {out }}\left(v_{4}\right) \preceq \mathcal{L}_{\text {out }}\left(v_{8}\right)$ and $\mathcal{L}_{i n}\left(v_{8}\right) \preceq \mathcal{L}_{i n}\left(v_{4}\right)$. Suppose the next to be visited is $v_{8}$, we can conclude that $v_{1} \rightsquigarrow v_{8}$. $\left(Q_{4}\right)$ consider $\operatorname{Reach}\left(v_{1}, v_{3}\right)$. We have $\mathcal{L}_{\text {out }}\left(v_{1}\right) \preceq \mathcal{L}_{\text {out }}\left(v_{3}\right)$ and $\mathcal{L}_{\text {in }}\left(v_{3}\right) \preceq \mathcal{L}_{\text {in }}\left(v_{1}\right)$, we cannot answer it by IP labels only, and DFS is needed. We start DFS from $v_{1}$, which has the two children $v_{4}$ and $v_{5}$. For $v_{4}$, we cannot do DFS further, because $v_{4} \not \hookrightarrow v_{3}$ for $\mathcal{L}_{\text {out }}\left(v_{4}\right) \succ \mathcal{L}_{\text {out }}\left(v_{3}\right)$ and $\mathcal{L}_{i n}\left(v_{3}\right) \succ \mathcal{L}_{i n}\left(v_{4}\right)$. For $v_{5}$, we cannot do DFS further neither, because $v_{5} \not \leftrightarrow v_{3}$ for $\mathcal{L}_{\text {out }}\left(v_{5}\right) \succ \mathcal{L}_{\text {out }}\left(v_{3}\right)$ and $\mathcal{L}_{\text {in }}\left(v_{3}\right) \succ \mathcal{L}_{\text {in }}\left(v_{5}\right)$.

The value of $p / q$ : Reconsider Eq. (4) (or Eq. (5)). Here, $p$ and $q$ imply either $|O u t(u)|$ and $|O u t(u) \cup \operatorname{Out}(v)|$ respectively, or $|\operatorname{In}(v)|$ and $|\operatorname{In}(v) \cup \operatorname{In}(u)|$ respectively. Since $p \leq q$, if $q-p$ is larger, $p / q$ becomes smaller, and $\operatorname{Pr}\left(\min _{k}\{\pi(\mathcal{A})\} \succ \min _{k}\{\pi(\mathcal{B})\}\right)$ becomes larger. Below, we show that the difference between $p$ and $q$ is not small. We discuss the case for $p=|O u t(u)|$, and $q=|\operatorname{Out}(u) \cup \operatorname{Out}(v)|$. The same can be applied to the $\operatorname{In}(v)$ and $\operatorname{In}(v) \cup \operatorname{In}(u)$.

First, as observed in many works, given a DAG $G$, the percentage of reachability queries $(\operatorname{Reach}(u, v))$ that are answered negatively, $u \ngtr v v$, over all possible reachability queries is over $90 \%$. On the other hand, the reachability queries that are answered positively, $u \rightsquigarrow v$, over all possible reachability queries is very small, which we call reachability-ratio (or simply R-ratio), denoted as $r$. We conducted testing to confirm $r$ is very small. We generate large DAGs by fixing the number of vertices to be 10 million vertices and increasing the average degree from 2 to 8 . For each DAG $G$, we sample 100 million vertex pairs, $u$ and $v$, to estimate the reachability ratio of $r$ for $G$. The R-ratio for the average degree $(2,3,4,5,6$, $7,8)$ is $(9.0 \mathrm{E}-7,6.2 \mathrm{E}-6,3.8 \mathrm{E}-5,2.17 \mathrm{E}-4,1.24 \mathrm{E}-3,5.68 \mathrm{E}-3,1.68 \mathrm{E}-$ 2). As a summary, for sparse DAGs, when the average degree $\leq 4$, the R-ratio is smaller than 0.0001, and for dense DAGs, when the average degree is 8 , the R-ratio is below 0.017 still.

Second, given the R-ratio $(r)$ for a DAG $G$, for two vertices $u$ and $v$, the expected value of $|O u t(u)|$ and $|O u t(v)|$ is $n r$, where $n$ is the number of vertices in $G$. If $u$ cannot reach $v$, the number of the common vertices between $\operatorname{Out}(u)$ and $O u t(v)$ is expected to be

$$
|O u t(u) \cap \operatorname{Out}(v)|=|\operatorname{Out}(u)| \times \frac{|O u t(v)|}{|V(G)|}=n r \cdot \frac{n r}{n}=n r^{2}
$$

Then, the percentage of $|O u t(u) \cap \operatorname{Out}(v)| /|\operatorname{Out}(u)|$ becomes $n r^{2} / n r=r$. This implies that $q$ is much larger than $p$ given $q=|\operatorname{Out}(u) \cup \operatorname{Out}(v)|$ and $p=|\operatorname{Out}(u)|$. This fact shows $\operatorname{Pr}\left(\min _{k}\{\pi(\mathcal{A})\} \succ \min _{k}\{\pi(\mathcal{B})\}\right)$ is large. 
The number of vertices to be visited by $D F S$ : Given $\operatorname{Reach}(u, v)$ and assume that we cannot answer by IP labels, and DFS is needed even though $u \not \downarrow \rightarrow v$. Suppose we visit a vertex $w$ by DFS. Because $u$ can reach $w$ by DFS, we have $|O u t(w)|<|O u t(u)|$ and $|\operatorname{In}(u)|<|\operatorname{In}(w)|$ due to $\operatorname{Out}(w) \subseteq \operatorname{Out}(u)$ and $\operatorname{In}(u) \subseteq$ $\operatorname{In}(w)$. While DFS along some path toward $v$ further, $\mid$ Out $(w) \mid$ will become significantly smaller than $|O u t(u)|$ and $|\operatorname{In}(u)|$ will become significantly smaller than $|\operatorname{In}(w)|$. Accordingly, the following is true.

$$
\begin{aligned}
\frac{|\operatorname{Out}(w)|}{|\operatorname{Out}(w) \cup \operatorname{Out}(v)|} & <\frac{|\operatorname{Out}(u)|}{|\operatorname{Out}(u) \cup \operatorname{Out}(v)|} \\
\frac{\operatorname{In}(v) \mid}{|\operatorname{In}(v) \cup \operatorname{In}(w)|} & <\frac{|\operatorname{In}(v)|}{|\operatorname{In}(v) \cup \operatorname{In}(u)|}
\end{aligned}
$$

As a result, if $u$ cannot reach $v$, during DFS, the vertex $w$ we visit will become more unlikely to reach $v$ by Eq. (4). Given this, the length of DFS is supposed not to large. We give a lemma below.

Lemma 4.1: Given Reach $(u, v)$, assume DFS is needed even though $u \nLeftarrow \rightarrow v$. Consider when a vertex $w$, as a descendant of $u$, is visited by DFS towards $v$. The followings are true.

$$
\begin{aligned}
\operatorname{Pr}\left(\mathcal{L}_{\text {out }}(u) \succ \mathcal{L}_{\text {out }}(v)\right) & <\operatorname{Pr}\left(\mathcal{L}_{\text {out }}(w) \succ \mathcal{L}_{\text {out }}(v)\right) \\
\operatorname{Pr}\left(\mathcal{L}_{\text {in }}(v) \succ \mathcal{L}_{\text {in }}(u)\right) & <\operatorname{Pr}\left(\mathcal{L}_{\text {in }}(v) \succ \mathcal{L}_{\text {in }}(w)\right)
\end{aligned}
$$

Proof Sketch: Since $w$ is a descendant of $u$, we have $|O u t(w)|<$ $|O u t(u)|$ and $|\operatorname{In}(u)|<|\operatorname{In}(w)|$ because $\operatorname{Out}(w) \subseteq O u t(u)$ and $\operatorname{In}(u) \subseteq \operatorname{In}(w)$. By Eq. (7) and Eq. (8), we have $\frac{|O u t(w)|}{|O u t(w) \cup O u t(v)|}$ $<\frac{|O u t(u)|}{|O u t(u) \cup O u t(v)|}$ and $\frac{\operatorname{In}(v) \mid}{|\operatorname{In}(v) \cup \operatorname{In}(w)|}<\frac{|\operatorname{In}(v)|}{|\operatorname{In}(v) \cup \operatorname{In}(u)|}$. By Eq. (4) (or its simplified version Eq. (5)), it shows that if $\frac{p}{q}$ decreases, $\operatorname{Pr}\left(\min _{k}\{\pi(\mathcal{A})\} \succ \min _{k}\{\pi(\mathcal{B})\}\right)$ will become larger accordingly. Therefore the probability of $\mathcal{L}_{\text {out }}(u) \succ \mathcal{L}_{\text {out }}(v)$ becomes smaller than the probability of $\mathcal{L}_{\text {out }}(w) \succ \mathcal{L}_{\text {out }}(v)$ and the probability of $\mathcal{L}_{i n}(v) \succ \mathcal{L}_{i n}(u)$ becomes smaller than the probability of $\mathcal{L}_{\text {in }}(v) \succ \mathcal{L}_{\text {in }}(w)$.

Lemma 4.1 suggests that the probability becomes higher for a descendant of $u, w$, to answer the reachability queries negatively. It implies that it is more likely to answer the reachability query by IP labels in DFS search

We analyze $\operatorname{Pr}\left(\mathcal{L}_{\text {out }}(u) \succ \mathcal{L}_{\text {out }}(v)\right)<\operatorname{Pr}\left(\mathcal{L}_{\text {out }}(w) \succ \mathcal{L}_{\text {out }}(v)\right)$ in Eq. (9), where the probability $\operatorname{Pr}(\cdot)$ is given as Eq. (5) for simplicity. Here, $w$ is a descendant of $u$. Let $p_{u}=|O u t(u)|$ and $q_{u}=\mid$ Out $(u) \cup$ Out $(v) \mid$. By Eq. $(5), \operatorname{Pr}\left(\mathcal{L}_{\text {out }}(u) \succ \mathcal{L}_{\text {out }}(v)\right) \approx$ $1-\left(p_{u} / q_{u}\right)^{k}$. Let $p_{w}=|\operatorname{Out}(w)|$ and $q_{w}=|\operatorname{Out}(w) \cup \operatorname{Out}(v)|$. By Eq. (7), we know $p_{w} / q_{w}<p_{u} / q_{u}$, and we denote $p_{w} / q_{w}$ as $\alpha \cdot p_{u} / q_{u}$ for $\alpha<1$. Hence, $\operatorname{Pr}\left(\mathcal{L}_{\text {out }}(w) \succ \mathcal{L}_{\text {out }}(v)\right) \approx$ $1-\alpha^{k} \cdot\left(p_{u} / q_{u}\right)^{k}$, which is significantly larger than $\operatorname{Pr}\left(\mathcal{L}_{\text {out }}(u) \succ\right.$ $\left.\mathcal{L}_{\text {out }}(v)\right)$. Furthermore, if we apply the same analysis to a vertex $\omega$ which is a child of $w$ in a similar. That is, Let $p_{\omega}=|O u t(\omega)|$ and $q_{\omega}=|\operatorname{Out}(\omega) \cup \operatorname{Out}(v)|$, and assume $p_{\omega} / q_{\omega}$ is $\beta \cdot p_{w} / q_{w}$ for $\beta<1$. Then, $\operatorname{Pr}\left(\mathcal{L}_{\text {out }}(\omega) \succ \mathcal{L}_{\text {out }}(v)\right) \approx 1-(\alpha \beta)^{k} \cdot\left(p_{u} / q_{u}\right)^{k}$. The same analysis can be applied to $\mathcal{L}_{i n}$. As discussed, while DFS search becomes deeper, it is much more likely to answer the reachability queries negatively, and therefore, it can stop in an early stage.

Reconsider $\left(Q_{4}\right)$ in Example 4.1 over $G$ as shown in Fig. 1(a), $\operatorname{Out}\left(v_{1}\right)=\left\{v_{1}, v_{4}, v_{5}, v_{6}, v_{7}, v_{8}, v_{9}, v_{10}, v_{11}\right\}, \operatorname{In}\left(v_{1}\right)=\left\{v_{1}\right\}$ and $\operatorname{Out}\left(v_{3}\right)=\left\{v_{3}, v_{4}, v_{6}, v_{7}, v_{8}, v_{9}\right\}, \operatorname{In}\left(v_{3}\right)=\left\{v_{0}, v_{3}\right\}$. Therefore, $\operatorname{Pr}\left(\mathcal{L}_{\text {out }}\left(v_{1}\right) \succ \mathcal{L}_{\text {out }}\left(v_{3}\right)\right)=\frac{1}{2}, \operatorname{Pr}\left(\mathcal{L}_{\text {in }}\left(v_{3}\right) \succ \mathcal{L}_{\text {in }}\left(v_{1}\right)\right)=$ $\frac{2}{3}$. The vertex $v_{1}$ has two children, $v_{4}$ and $v_{5}$. Suppose it conducts DFS to either of them. $\operatorname{Pr}\left(\mathcal{L}_{\text {out }}\left(v_{4}\right) \succ \mathcal{L}_{\text {out }}\left(v_{3}\right)\right)=\frac{14}{15}$, $\operatorname{Pr}\left(\mathcal{L}_{\text {in }}\left(v_{3}\right) \succ \mathcal{L}_{\text {in }}\left(v_{4}\right)\right)=\frac{9}{10}$ and $\operatorname{Pr}\left(\mathcal{L}_{\text {out }}\left(v_{5}\right) \succ \mathcal{L}_{\text {out }}\left(v_{3}\right)\right)=$ $\frac{125}{126}, \operatorname{Pr}\left(\mathcal{L}_{i n}\left(v_{3}\right) \succ \mathcal{L}_{i n}\left(v_{5}\right)\right)=\frac{5}{6}$. The probability becomes larger while DFS search.
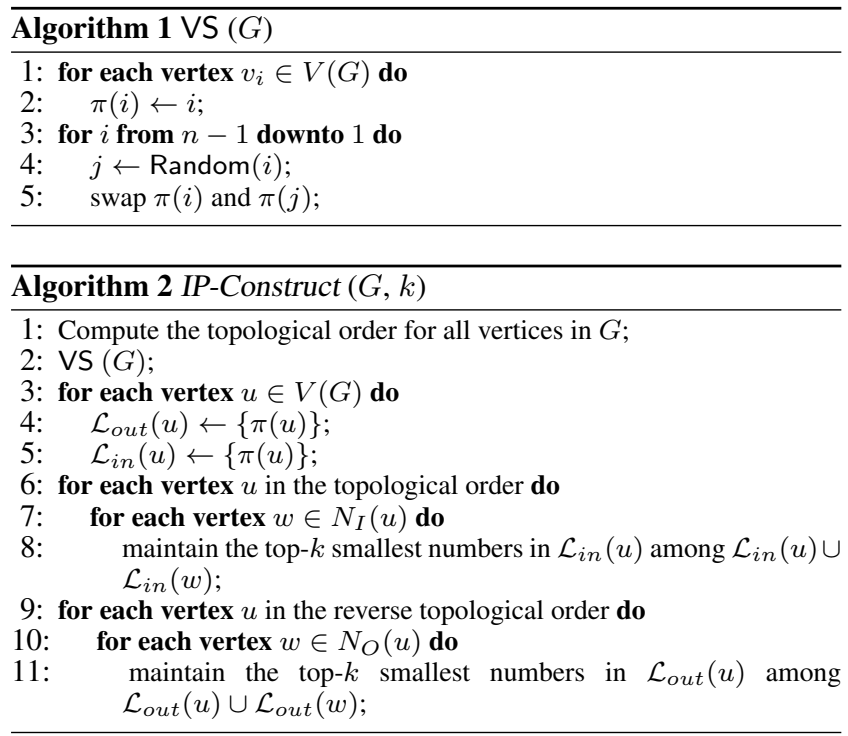

\section{COMPUTING IP LABELS}

We discuss computing IP. First, we discuss the algorithm to compute a permutation $(\pi)$, which assigns every $u \in V(G)$ a unique permutation number $\pi(u)$ in $O(n)$. Then, we give an algorithm to compute IP based on the permutation in $O(k(m+n))$.

\subsection{Computing Permutation}

To compute a permutation, we adopt the Knuth shuffle algorith$\mathrm{m}$ [22], denoted as KS, which is also known as the Fisher-Yates shuffle algorithm [15]. The KS algorithm is unbiased, and has the equal chance to generate every possible permutation, which meets the requirement for min-wise independent permutation technique. It is important to us that the complexity of the algorithm is $O(n)$. We show our VS algorithm in Algorithm 1, which is based on the $\mathrm{KS}$ algorithm. The VS algorithm first assigns an initial permutation number in $[0, n-1]$ to every vertex $v_{i}$ in $G$, where $n=|V(G)|$ (line 1-2). Then, in a for loop (line 3-5), from $i=n-1$ to 1 , it generates a random number $j$ uniformly in the range of $[0, i]$ (line 4 ), and swaps $\pi(i)$ and $\pi(j)$ between $i$ and $j$ (line 5). The time complexity of the VS algorithm is $O(n)$. We can see that the probability of $\pi(i)=x$, for any $i, x \in[0, n-1]$, is equal to the probability that $x$ has not been randomly chosen for exchange in the first $n-i-1$ iterations multiplies the probability that $x$ is chosen for exchange in the $(n-i)$-th iteration. That is,

$$
\operatorname{Pr}(\pi(i)=x)=\frac{n-1}{n} \times \frac{n-2}{n-1} \times \cdots \frac{i+1}{i+2} \times \frac{1}{i+1}=\frac{1}{n}
$$

Therefore, for any $i \in[0, n-1], \pi(i)$ has the equal chance to be any value in $[0, n-1]$. The VS algorithm (Algorithm 1 ) is equally likely to generate any possible independent permutation.

\subsection{IP Computing}

We discuss how to compute IP. Consider a vertex $u$, its $\mathcal{L}_{\text {out }}(u)$ can be computed by all $\mathcal{L}_{\text {out }}(w)$ in its out-neighbors $\left(\in N_{O}(u)\right)$. This is because $\operatorname{Out}(u)=\bigcup_{w \in N_{O}(u)} \operatorname{Out}(w)$. Therefore, $\mathcal{L}_{\text {out }}(u)$ must be the subset of $\bigcup_{w \in N_{O}(u)} \mathcal{L}_{\text {out }}(w)$. In other words, $\mathcal{L}_{\text {out }}(u)$ consists of the top- $k$ smallest numbers of $\bigcup_{w \in N_{O}(u)} \mathcal{L}_{\text {out }}(w)$. In a similar way, its $\mathcal{L}_{i n}(u)$ can be computed by all $\mathcal{L}_{i n}(w)$ in its inneighbors $\left(\in N_{I}(u)\right)$. This is because $\operatorname{In}(u)=\bigcup_{w \in N_{I}(u)} \operatorname{In}(w)$. Therefore, $\mathcal{L}_{i n}(u)$ must be the subset of $\bigcup_{w \in N_{I}(u)} \mathcal{L}_{i n}(w)$. In 
other words, $\mathcal{L}_{i n}(u)$ consists of the top- $k$ smallest numbers from $\bigcup_{w \in N_{I}(u)} \mathcal{L}_{i n}(w)$. Based on this idea, we design an algorithm called IP-Construct to compute IP labels for vertices in $G$.

The IP-Construct is shown in Algorithm 2. First, we compute the topological order for all vertices in $G$ (line 1), and compute the permutation $(\pi)$ by calling the VS algorithm (line 2). First, for every vertex $u \in V(G)$, it assigns $\pi(u)$ in $\mathcal{L}_{\text {out }}(u)$ and $\mathcal{L}_{\text {in }}(u)$ (line 35). Below, we maintain $\mathcal{L}_{\text {out }}(u)$ and $\mathcal{L}_{\text {in }}(u)$ in ascending order. It is worth mentioning that the size of $\mathcal{L}_{\text {out }}(u)$ and the size of $\mathcal{L}_{\text {in }}(u)$ is up to $k$. Second, we visit vertices following the topological order (line 6-8), and compute $\mathcal{L}_{i n}(u)$ from its in-neighbors (line 7-8). To update $\mathcal{L}_{i n}(u)$ by one additional in-neighbor $w$, it selects the top- $k$ smallest numbers from two lists, $\mathcal{L}_{i n}(u)$ and $\mathcal{L}_{i n}(w)$, which are both sorted in ascending order. Finally, we visit vertices following the reverse topological order (line 9-11), and compute $\mathcal{L}_{\text {out }}(u)$ from its out-neighbors (line 10-11) in a similar way.

Construction time and Index size: To compute IP by Algorith$\mathrm{m} 2$, it takes $O(m+n)$ to compute the topological order (line 1), and $O(n)$ to compute the permutation (Algorithm 1) (line 2). The initialization of $\mathcal{L}_{\text {out }}(u)$ and $\mathcal{L}_{\text {in }}(u)$ for every $u \in G$ (line 3-5) is $O(n)$. Then, it takes $O(k(n+m))$ time to compute $\mathcal{L}_{i n}(u)$ for every $u \in G$ (line 6-8). This is because it takes $O(k)$ to update a vertex $u$ 's $\mathcal{L}_{i n}(u)$ using $\mathcal{L}_{i n}(w)$ for an in-neighbor $w$ of $u$ (line 8) by merging the two sorted lists of size up to $k$, and such update is to be done for every of $m$ edges when accessing all $n$ vertices in $G$. In a similar way, it takes $O(k(n+m))$ time to compute $\mathcal{L}_{\text {out }}(u)$ for every $u \in G$ in line 11 . Overall, the time complexity is $O(k(n+m))$. Our algorithm is more efficient than GRAIL even though both are $O(k(n+m))$. In our algorithm, we only need to scan $G$ twice. In GRAIL, it needs to scan $G k$ times to generate $k$ spanning trees. The IP index size is at most $2 k n$, because $\left|\mathcal{L}_{\text {out }}(u)\right| \leq k$ and $\left|\mathcal{L}_{\text {in }}(u)\right| \leq k$ for every $u \in G$. Comparing with GRAIL, our index size is up to $2 k n$, whereas the index size for GRAIL is $2 k n$.

\section{TWO ADDITIONAL LABELS}

In this section, we discuss two additional labels together to be used with IP labels. The two additional labels are used to reduce the DFS search cost.

\subsection{A Level Label}

In order to early stop in DFS for answering $\operatorname{Reach}(u, v)$, we introduce a level label for every vertex $u$, denoted as $\mathcal{L}_{\text {level }}(u)$. The $\mathcal{L}_{\text {level }}(u)$ consists of two parts, $\mathcal{L}_{\text {up }}(u)$ and $\mathcal{L}_{\text {down }}(u)$, and are defined in Eq. (10) and Eq. (11).

$$
\begin{aligned}
\mathcal{L}_{\text {up }}(u) & = \begin{cases}0 & \text { if }\left|N_{O}(u)\right|=0 \\
1+\max _{v \in N_{O}(u)}\left\{\mathcal{L}_{\text {up }}(v)\right\} & \text { otherwise }\end{cases} \\
\mathcal{L}_{\text {down }}(u) & = \begin{cases}0 & \text { if }\left|N_{I}(u)\right|=0 \\
1+\max _{v \in N_{I}(u)}\left\{\mathcal{L}_{\text {down }}(v)\right\} & \text { otherwise }\end{cases}
\end{aligned}
$$

Since we have already computed the topological order, it takes $O(n+m)$ to compute the level labels for vertices in $G$. Based on Eq. (10) and Eq. (11), we have the following theorem about the level labels, which helps to prune unnecessary search paths in DFS effectively.

Theorem 6.3: Given two vertices $u$ and $v$ in $G$. If $u$ can reach $v$, for $u \neq v$, then both $\mathcal{L}_{u p}(u)>\mathcal{L}_{\text {up }}(v)$ and $\mathcal{L}_{\text {down }}(u)<$ $\mathcal{L}_{\text {down }}(v)$ must be true.

Proof Sketch: Based on Eq. (10), $\mathcal{L}_{u p}(u)$ must be larger than $\mathcal{L}_{u p}(w)$ for $w \in N_{O}(u)$, and be larger than any descendant of

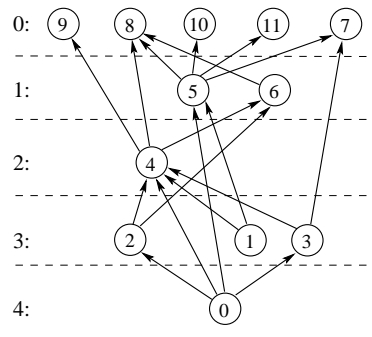

(a) $\mathcal{L}_{u p}(u)$ for $u \in G$

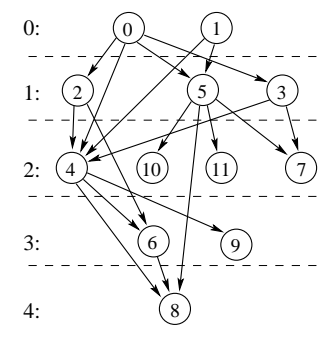

(b) $\mathcal{L}_{\text {down }}(u)$ for $u \in G$
Figure 2: Level Labels for $G$ in Fig. 1(a)

$u$. If $u$ can reach $v$, then $N_{O}(u) \neq \emptyset$ and $v$ is a descendant of $u$. This leads to the conclusion that $\mathcal{L}_{u p}(u)>\mathcal{L}_{u p}(v)$. Also, based on Eq. (11), $\mathcal{L}_{\text {down }}(v)$ must be larger than $\mathcal{L}_{\text {down }}(\omega)$ for $\omega \in N_{I}(u)$, and be larger than any ascendant of $v$. If $u$ can reach $v$, then $N_{I}(v) \neq \emptyset$ and $u$ is an ascendant of $v$. This leads to the conclusion that $\mathcal{L}_{\text {down }}(u)<\mathcal{L}_{\text {down }}(v)$.

Example 6.2: Consider graph $G$ in Fig. 1(a), the level labels of $G$ are shown in Fig. 2. Consider $\operatorname{Reach}\left(v_{1}, v_{0}\right)$ and $\operatorname{Reach}\left(v_{1}, v_{2}\right)$. As shown in Fig. 2(a), $v_{1}$ cannot reach $v_{0}$ nor $v_{2}$, because $\mathcal{L}_{u p}\left(v_{0}\right)=$ 4 and $\mathcal{L}_{u p}\left(v_{2}\right)=3$ are not smaller than $\mathcal{L}_{u p}\left(v_{1}\right)=3$. Consider Reach $\left(v_{3}, v_{5}\right)$. From Fig. 2(a), it seems $v_{3}$ can reach $v_{5}$, because $\mathcal{L}_{\text {up }}\left(v_{3}\right)>\mathcal{L}_{\text {up }}\left(v_{5}\right)$. However, From Fig. 2(b), we have $\mathcal{L}_{\text {down }}\left(v_{5}\right)=\mathcal{L}_{\text {down }}\left(v_{3}\right)$. Therefore, $v_{3}$ cannot reach $v_{5}$.

The level labels are effective as we will show in our experimental study, but are not new. GRAIL [30] uses a similar technique like $\mathcal{L}_{\text {up }}(u)$, and TF-Label [10] uses $\mathcal{L}_{\text {down }}(u)$. The difference between the TF-Label and ours, in terms of $\mathcal{L}_{\text {down }}(u)$ is as follows. We use $\mathcal{L}_{\text {down }}(u)$ to early stop in DFS at run-time, whereas TF-Label uses to construct 2-Hop labels.

\subsection{A Huge-Vertex Label}

A key factor that affects the performance of DFS at run-time is how to deal with those vertices that have large out-degree. We call such a vertex as a huge-vertex denoted as HV. With the existence of such huge-vertices, in particular, in power-law graphs [14], when it performs DFS starting from a huge-vertex at run-time, the time consumption will become large, because its out-degree is high, and the size of its out-neighbors is large. There are too many possible paths to search in DFS. Take Reach $\left(v_{0}, v_{11}\right)$ as an example, and consider DFS search of $G$ in Fig. 1(a). $v_{0}$ itself is a relative huge vertex in comparison with other vertices in this small graph $G$, so as two of its children $v_{4}$ and $v_{5}$. If the DFS search order is $\left(v_{0}, v_{2}\right.$, $\left.v_{6}, v_{8}, v_{4}, v_{9}, v_{3}, v_{7}, v_{5}, v_{10}, v_{11}\right)$, then it will search nearly the entire $G$ before finally answering this reachability query. There are certain huge-vertices in many real graphs that we need to deal with to reduce the run-time cost.

We propose a new simple but effective label, called HV-Label, and denoted as $\mathcal{L}_{h v}(v)$ for every vertex $v \in G$. Here, $\mathcal{L}_{h v}(v)$ contains up to top- $h$ largest $H V$ vertices, $\{u\}$, if $u$ can reach $v$ and the out-degree of $u$ is larger than $\mu$ where $n=|V(G)|$ and $\mu$ is a user-given threshold, such that $\mathcal{L}_{h v}(v)=\left\{u \mid u \rightsquigarrow v \wedge d_{O}(u)>\right.$ $\mu\}$ for $\left|\mathcal{L}_{h v}(u)\right| \leq h$.

Answering with HV-Label: With HV-Label, we can decide $w \rightsquigarrow$ $v$, if $w \in \mathcal{L}_{h v}(v)$. In other words, suppose that we DFS search to a huge-vertex $w$ for answering $\operatorname{Reach}(u, v)$. If $w \in \mathcal{L}_{h v}(v)$, then we can answer $u \rightsquigarrow v$ immediately without any needs of DFS. If $w$ is not a huge-vertex of $v\left(w \notin \mathcal{L}_{h v}(v)\right)$ and $\left|\mathcal{L}_{h v}(v)\right|<h$, $w \not \rightarrow v$. In addition, if $w$ is a huge-vertex but $w \notin \mathcal{L}_{h v}(v)$ and $d_{O}(w)$ is larger than some out-degree of the vertices in $\mathcal{L}_{h v}(v)$, we 


\begin{tabular}{|c||l||c|l|}
\hline Vertex & $\mathcal{L}_{h v}\left(v_{i}\right)$ & Vertex & $\mathcal{L}_{h v}\left(v_{i}\right)$ \\
\hline \hline$v_{0}$ & $\{0\}$ & $v_{6}$ & $\{0,4\}$ \\
\hline$v_{1}$ & $\emptyset$ & $v_{7}$ & $\{0,5\}$ \\
\hline$v_{2}$ & $\{0\}$ & $v_{8}$ & $\{0,5\}$ \\
\hline$v_{3}$ & $\{0\}$ & $v_{9}$ & $\{0,4\}$ \\
\hline$v_{4}$ & $\{0,4\}$ & $v_{10}$ & $\{0,5\}$ \\
\hline$v_{5}$ & $\{0,5\}$ & $v_{11}$ & $\{0,5\}$ \\
\hline
\end{tabular}

Table 3: Huge-Vertices for $G$ shown in Fig. 1(a)

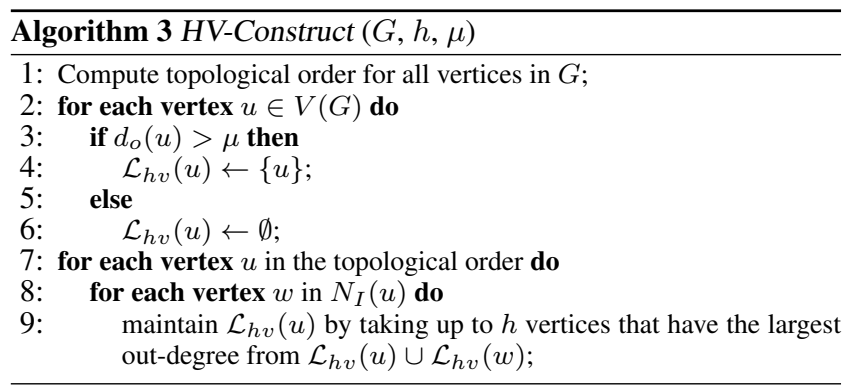

can answer $w \not \leftrightarrow v$ by definition.

Example 6.3: Consider $G$ shown in Fig. 1(a), where $n=12$. Let $\mu=2$ and $h=2$. A vertex is a huge-vertex if its out-degree is larger than 2. For $G$ shown in Fig. 1(a), the huge-vertices are $v_{0}, v_{4}$ and $v_{5}$. The $H V$-Labels computed for $G$ are shown in Table 3 . Consider a reachability query $\operatorname{Reach}\left(v_{0}, v_{11}\right)$. Here, $v_{0}$ is a huge-vertex and $0 \in \mathcal{L}_{h v}\left(v_{11}\right)$, we know $v_{0} \rightsquigarrow v_{11}$. Consider another reachability query $\operatorname{Reach}\left(v_{0}, v_{1}\right)$. We can answer $v_{0} \not v_{1}$, because $v_{0}$ is a huge-vertex and $\mathcal{L}_{h v}\left(v_{1}\right)$ is empty. Also, we can answer Reach $\left(v_{5}, v_{6}\right)$ as $v_{5} \not v_{6}$ using $H V$-Labels. Here, $v_{5}$ is a huge-vertex and $\mathcal{L}_{h v}\left(v_{6}\right)=\{0,4\}$. By $H V$-Label, we know that $5 \notin \mathcal{L}_{h v}\left(v_{6}\right)$ but the out-degree of $v_{5}$ is larger than the out-degree of $v_{4}$, and therefore, the conclusion of $v_{5} \not v_{6}$ can be made.

The algorithm to compute HV-Labels for a graph $G$ is given Algorithm 3, called HV-Construct. The idea to construct HV-Labels is very similar to the IP-Construct algorithm. The time complexity for Algorithm 3 is $O(h(n+m))$ and the space is $O(h n)$.

We discuss the two user-given thresholds $h$ and $\mu$ below. Here, $h$ determines the size of $H V$-Label, $\mathcal{L}_{h v}(u)$, for every vertex in $G$, and $\mu$ determines what vertices are huge-vertices to be selected. Since $h$ is for a few of huge-vertices, it does not need to be large. Decreasing $\mu$ will make more vertices to be huge-vertices. Doing so will include really huge-vertices in $H V$-Label. However, it is not necessary to use a small $\mu$. We suggest to use $\mu=100$ after many testings. In other words, a vertex is a huge-vertices if its out-degree is $>100$.

\section{ANSWER Reach $(u, v)$}

We have discuss three labels, IP labels, Level labels, and $H V$ Label. For every vertex $u \in G$, the labels are $\mathcal{L}_{\text {out }}(u), \mathcal{L}_{\text {in }}(u)$, $\mathcal{L}_{\text {up }}(u), \mathcal{L}_{\text {down }}(u)$, and $\mathcal{L}_{\text {hv }}(u)$. Here, both $\mathcal{L}_{\text {out }}(u)$ and $\mathcal{L}_{\text {in }}(u)$ are up to $k$ numbers, $\mathcal{L}_{u p}(u)$ and $\mathcal{L}_{\text {down }}(u)$ are fixed to 2 numbers, and $\mathcal{L}_{h v}(u)$ is up to $h$ numbers. In total, the max size of labels are $2 k+h+2$ for a vertex.

We process a reachability query $\operatorname{Reach}(u, v)$ in a way as given in the IP+ algorithm (Algorithm 4). First, it checks the trivial case whether $u=v$, and answers $u \rightsquigarrow v$ if $u=v$ (line 1). Second, it checks IP labels, and answers $u \not \hookleftarrow v$ if either $\mathcal{L}_{\text {out }}(u) \succ \mathcal{L}_{\text {out }}(v)$ is true or $\mathcal{L}_{i n}(v) \succ \mathcal{L}_{i n}(u)$ is true (line 2). Third, it checks Level labels, and answers $u \not \leftrightarrow v$ if either $\mathcal{L}_{u p}(u) \leq \mathcal{L}_{u p}(v)$ is true or

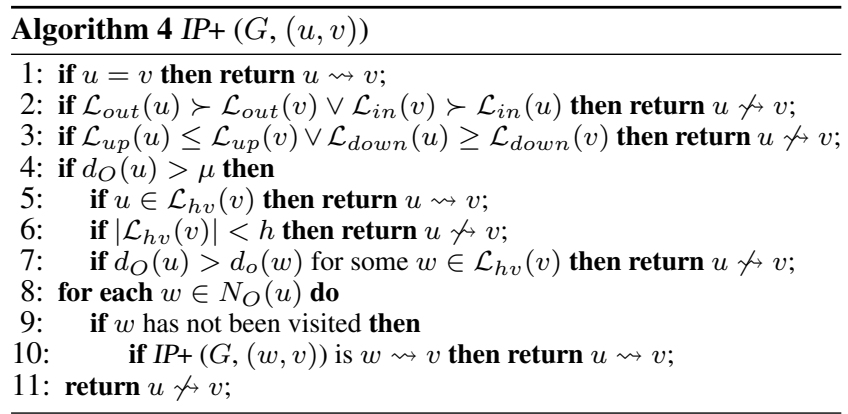

\begin{tabular}{|l||r|r|r|r|}
\hline Dataset & $|V(G)|$ & $|E(G)|$ & $d_{\text {avg }}$ & R-ratio $(r)$ \\
\hline \hline citeseer & 693,947 & 312,282 & 0.450 & $3.20 \mathrm{E}-6$ \\
\hline email & 231,000 & 223,004 & 0.965 & $5.06 \mathrm{E}-2$ \\
\hline LJ & 971,232 & $1,024,140$ & 1.054 & $2.13 \mathrm{E}-1$ \\
\hline mapped-100K & $2,658,702$ & $2,660,628$ & 1.000 & $1.56 \mathrm{E}-6$ \\
\hline mapped-1M & $9,387,448$ & $9,440,404$ & 1.005 & $7.00 \mathrm{E}-7$ \\
\hline uniprotenc22m & $1,595,444$ & $1,595,442$ & 0.999 & $1.45 \mathrm{E}-6$ \\
\hline uniprotenc100m & $16,087,295$ & $16,087,293$ & 0.999 & $1.60 \mathrm{E}-7$ \\
\hline uniprotenc150m & $25,037,600$ & $25,037,598$ & 0.999 & $1.30 \mathrm{E}-7$ \\
\hline web & 371,764 & 517,805 & 1.392 & $1.48 \mathrm{E}-1$ \\
\hline wiki & $2,281,879$ & $2,311,570$ & 1.013 & $8.14 \mathrm{E}-3$ \\
\hline yago & $16,375,503$ & $25,908,132$ & 1.582 & $1.00 \mathrm{E}-6$ \\
\hline twitter & $18,121,168$ & $18,359,487$ & 1.013 & $7.39 \mathrm{E}-2$ \\
\hline web-uk & $22,753,644$ & $38,184,039$ & 1.678 & $1.50 \mathrm{E}-1$ \\
\hline \hline citeseerx & $6,540,399$ & $15,011,259$ & 2.295 & $4.07 \mathrm{E}-4$ \\
\hline patent & $3,774,768$ & $16,518,947$ & 4.376 & $2.36 \mathrm{E}-3$ \\
\hline go-uniprot & $6,967,956$ & $34,770,235$ & 4.990 & $3.64 \mathrm{E}-6$ \\
\hline govwild & $8,022,880$ & $23,652,610$ & 2.948 & $7.20 \mathrm{E}-5$ \\
\hline dbpedia & $3,365,623$ & $7,989,191$ & 2.374 & $2.47 \mathrm{E}-2$ \\
\hline HostLink & $12,754,590$ & $26,669,293$ & 2.091 & $4.48 \mathrm{E}-2$ \\
\hline
\end{tabular}

Table 4: Large Real Graphs

$\mathcal{L}_{\text {down }}(u) \geq \mathcal{L}_{\text {down }}(v)$ is true (line 3 ). Forth, it uses HV-Label to answer in line (4-7) when $u$ is a huge-vertex $\left(d_{O}(u)>\mu\right)$. There are three cases. (a) It answers $u \rightsquigarrow v$ if the huge-vertex $u$ is maintained in $\mathcal{L}_{h v}(v)$ (line 5). (b) It answers $u \not p v$ if $\left|\mathcal{L}_{h v}(v)\right|<h$ (line 6), where the condition $u \notin \mathcal{L}_{h v}(v)$ is true due to line 5. (c) It answers $u \not \downarrow v$ if the out-degree of $u$ is larger than the out-degree of some vertex in $\mathcal{L}_{h v}(v)$ (line 7). Fifth, if none of the above is true, it will conducts DFS to search the vertices that have not been visited before (line 8-10). Finally, it will answer $u \not \iota \rightarrow v$ if it is impossible to answer positively.

Query Time: We discuss the time complexity of Algorithm 4 for answering $\operatorname{Reach}(u, v)$. The time complexity is $O(k)$, because both labels used are sorted, when IP labels over $u$ and $v$ can be directly used to answer the reachability query without the needs of DFS. Next, we consider the cases when DFS is needed. First, suppose the answer of $\operatorname{Reach}(u, v)$ is $u \rightsquigarrow v$. Then, the time complexity is related to the vertices that can be possibly on some paths from $u$ to $v$. The number of such vertices is $|\operatorname{Out}(u) \cap \operatorname{In}(v)|$, which is $n r^{2}$, in a similar way as obtained in Eq. (6), where $n$ is the number of vertices and $r$ is the R-ratio of the graph $G$. Since it consumes $O(k)$ in every vertex visited, the time complexity is $O\left(k n r^{2}\right)$. It does not take the HV-Label into consideration, which can be effectively used to reduce the query time. Second, suppose the answer of $\operatorname{Reach}(u, v)$ is $u \not \sim v$. As discussed above, the IP labels can be effectively used to terminate DFS with the assistance of Level labels and HV-Label during DFS. The DFS search will be terminated after a small number of vertices to be visited, which we consider as a constant (refer to Eq. (9) in Lemma 4.1). Such a number is much smaller than $n$ and it is not related to $m$. It is much less than but can be bounded by $O\left(k n r^{2}\right)$. 


\begin{tabular}{|c|c|c|c|c|c|c|c|c|c|}
\hline Dataset & GRAIL & GRAIL* & ScaGRAIL & PWAH8 & TF-Label & $H L$ & $D L$ & Ferrari- $G$ & $I P+$ \\
\hline citeseer & 0.959 & 0.635 & 2.611 & 0.530 & 0.780 & 1.108 & 0.589 & 0.537 & 0.37 \\
\hline email & 0.303 & 0.166 & 1.067 & 0.225 & 0.113 & 0.289 & 0.147 & 0.194 & 0.09 \\
\hline LJ & 1.299 & 0.695 & 5.367 & 1.052 & 0.628 & 1.319 & 0.667 & 0.887 & 0.54 \\
\hline mapped-100K & 3.106 & 2.032 & 9.231 & 0.527 & 2.307 & 4.422 & 2.272 & 2.748 & 1.64 \\
\hline mapped-1M & 12.334 & 7.299 & 35.229 & 2.830 & 13.049 & 17.347 & 7.736 & 9.986 & 6.12 \\
\hline uniprotenc $22 \mathrm{~m}$ & 2.451 & 1.206 & 6.790 & 1.485 & 2.223 & 2.220 & 1.038 & 0.919 & $\overline{0.84}$ \\
\hline uniprotenc100m & 33.214 & 13.658 & 90.518 & 17.543 & 39.848 & 26.297 & 12.889 & 14.022 & 12.46 \\
\hline uniprotenc150m & 58.242 & 23.820 & 145.576 & 29.169 & 58.529 & 44.712 & 22.280 & 24.292 & 18.96 \\
\hline web & 0.495 & 0.340 & 1.900 & 0.740 & 0.454 & 0.717 & 0.370 & 0.449 & 0.25 \\
\hline wiki & 2.886 & 1.329 & 7.335 & 0.459 & 1.011 & 2.878 & 1.375 & 1.927 & 1.22 \\
\hline yago & 27.489 & 21.353 & 85.837 & 12.723 & 16.905 & 40.886 & 21.121 & 35.977 & 13.35 \\
\hline twitter & 32.323 & 13.162 & - & 11.864 & 15.291 & 27.645 & 13.719 & 19.972 & 12.44 \\
\hline web-uk & 44.031 & 17.854 & - & 248.814 & - & 1081.390 & 24.240 & 26.927 & 17.46 \\
\hline citeseerx & 23.170 & 23.730 & 61.188 & 19.976 & 91.877 & 131.962 & 12.045 & 19.792 & $\overline{7.54}$ \\
\hline patent & 21.404 & 23.498 & 82.217 & 1098.786 & 238.422 & - & 135.628 & 41.729 & 9.00 \\
\hline go-uniprot & 44.557 & 39.342 & 70.277 & 38.668 & 64.501 & 27.591 & 18.277 & 40.365 & 9.68 \\
\hline govwild & 29.237 & 28.300 & 62.963 & 30.520 & 139.247 & 32.230 & 18.584 & 19.924 & 8.45 \\
\hline dbpedia & 17.503 & 16.099 & 45.570 & 7.771 & 12.152 & 10.926 & 4.741 & 6.236 & 3.75 \\
\hline HostLink & 58.279 & 63.388 & 158.537 & 42.759 & 303.723 & 33.533 & 16.815 & 24.760 & 11.89 \\
\hline
\end{tabular}

Table 5: Index Construction Time on Large Real Graphs (in second)

\begin{tabular}{|c|c|c|c|c|c|c|c|c|c|}
\hline Dataset & GRAIL & GRAIL $^{*}$ & ScaGRAIL & PWAH8 & TF-Label & HL & $D L$ & Ferrari-G & $I P+$ \\
\hline citeseer & 10.588 & 18.530 & 11.941 & 6.565 & 1.789 & 7.713 & 7.145 & 13.607 & 9.440 \\
\hline email & 3.525 & 6.168 & 4.365 & 2.342 & 0.851 & 2.693 & 2.586 & 5.792 & 3.664 \\
\hline LJ & 14.820 & 25.935 & 18.785 & 9.644 & 3.956 & 11.677 & 11.227 & 18.096 & 19.112 \\
\hline mapped-100K & 40.569 & 70.995 & 51.112 & 10.715 & 17.185 & 39.276 & 34.424 & 30.766 & 53.899 \\
\hline mapped-1M & 143.241 & 250.672 & 180.650 & 38.078 & 73.933 & 137.352 & 116.089 & 109.546 & 171.253 \\
\hline uniprotenc $22 \mathrm{~m}$ & 24.345 & 42.603 & 30.675 & 18.635 & 6.294 & 18.620 & 18.467 & 45.646 & 24.494 \\
\hline uniprotenc100m & 245.473 & 429.577 & 310.668 & 208.622 & 76.721 & 204.739 & 197.182 & 460.261 & 251.150 \\
\hline uniprotenc150m & 382.043 & 668.576 & 486.188 & 349.251 & 131.920 & 336.998 & 318.504 & 716.331 & 394.966 \\
\hline web & 5.672 & 9.927 & 7.615 & 4.330 & 2.560 & 5.894 & 4.848 & 8.269 & 6.952 \\
\hline wiki & 34.818 & 60.933 & 43.940 & 8.987 & 8.858 & 26.325 & 26.203 & 26.622 & 51.992 \\
\hline yago & 249.870 & 437.273 & 315.017 & 134.646 & 98.831 & 290.393 & 223.766 & 312.701 & 250.567 \\
\hline twitter & 276.507 & 483.887 & - & 95.566 & 70.111 & 211.143 & 202.480 & 237.106 & 384.894 \\
\hline web-uk & 347.193 & 607.587 & - & 260.100 & - & 4520.436 & 697.646 & 458.302 & 442.890 \\
\hline citeseerx & 249.497 & 399.194 & 303.017 & 148.781 & 1523.491 & 1531.189 & 1117.542 & 186.650 & $\overline{c 151.018}$ \\
\hline patent & 143.996 & 230.393 & 250.212 & 5334.114 & 4731.991 & - & 625.282 & 205.195 & $\mathbf{1 3 7 . 8 6 5}$ \\
\hline go-uniprot & 265.806 & 425.290 & 399.486 & 244.026 & 430.527 & 291.748 & 247.852 & 378.774 & 184.683 \\
\hline govwild & 306.049 & 489.678 & 409.899 & 304.061 & 3122.743 & 319.489 & 191.040 & 402.808 & 193.963 \\
\hline dbpedia & 128.388 & 205.421 & 159.639 & 59.897 & 52.189 & 85.040 & 53.299 & 106.202 & 94.275 \\
\hline HostLink & 486.549 & 778.478 & 580.031 & $\mathbf{1 1 5 . 4 3 5}$ & 5670.271 & 269.332 & 201.445 & 229.391 & 369.603 \\
\hline
\end{tabular}

Table 6: Index Size of Large Real Graphs (in MB)

\section{EXPERIMENTAL STUDIES}

We conduct extensive experimental studies, and report our findings in this section. We denote our approach as IP+ (Algorithm 2), which uses IP labels with two additional Level labels and $H V$ Label. And we compare IP+ with the state-of-the-art reachability approaches including GRAIL [29], GRAIL ${ }^{*}$ [30], ScaGRAIL [17, 29], PWAH8 [27], TF-Label [10], HL [19], DL [19], and Ferrar$i$ [24]. Here, GRAIL and Ferrari are two state-of-art Label+ $G$ approaches, and their index size and construction time are in linear. We test Ferrari using the Ferrari-G index given in [24], because Ferrari-G is scalable to handle massive-scale graphs. GRAIL* is the improved version of GRAIL [30] and ScaGRAIL is the implementation of GRAIL in the SCARAB framework [17]. ${ }^{1}$ We also test the Label-Only approaches. PWAH8 is the state-of-the-art transitive closure compression approach. And TF-Label [10], HL [19], and $D L[19]$ are the three state-of-the-art Label-Only approaches based on 2-Hop labels. We use the source codes provided by the authors to test the existing approaches. The source code of all approaches is implemented in $\mathrm{C}++$ and compiled by $\mathrm{G}++$ 4.8.1. All

${ }^{1}$ We can also implement our $I P+$ in the SCARAB framework. We will leave it as our future work. experiments are performed on machine with $2.67 \mathrm{GHz}$ Intel Xeon X5550 CPU, 24GB RAM and running Linux.

The three measures of the testing are: index construction time, index size, and query time. Programs that run $\geq 24$ hours or exceed the memory limit (24GB) will be terminated, and the results will be shown "- " in the tables or "INF" in the figures.

Real Datasets: We use all large datasets used in the recent works [29, 30, 17, 27, 10, 19, 24]. Here, citeseer, citeseerx (citeseerx. ist.psu.edu), and patent (snap. stanford.edu) are 3 citation networks in which the out-degree of the non-leaf vertices is about 10 to 30. go-uniprot is the joint graph of Gene Ontology terms with the annotations file from the the universal protein resource database UniProt (www . uniprot . org). uniprotenc22m, uniprotenc $100 \mathrm{~m}$, and uniprotenc $150 \mathrm{~m}$ are subgraphs of the complete RDF graph of UniProt. mapped-100K and mapped-1M are two datasets used in $[17,19]$. email is a DAG of the communication network email-EuAll. LJ is a DAG of social network socLiveJournal1. web is the DAG of web graph web-Google. wiki is a DAG of wiki-talk, got from Wikipedia. These 4 datasets are from snap. stanford. edu. govwild is a large RDF graph from govwild.hpi-web. de and is transformed into the corresponding DAG. yago is a DAG of a large RDF representing a knowledge 


\begin{tabular}{|c|c|c|c|c|c|c|c|c|c|}
\hline Dataset & GRAIL & GRAIL ${ }^{*}$ & ScaGRAIL & PWAH8 & TF-Label & $H L$ & $D L$ & Ferrari-G & $I P+$ \\
\hline citeseer & 363.738 & 96.722 & 108.564 & 138.810 & 7.869 & 60.558 & $\overline{59.351}$ & 67.833 & 24.503 \\
\hline email & 12495.148 & 111.984 & 150.430 & 165.464 & 19.341 & 38.145 & 36.005 & 101.753 & 38.135 \\
\hline LJ & 4267759 & 216.862 & 1141.400 & 171.929 & 66.592 & 65.465 & 65.049 & 94.538 & 86.988 \\
\hline mapped-1M & 413.987 & 225.407 & 79.515 & 65.853 & 99.754 & 106.026 & 103.001 & 27.083 & 44.186 \\
\hline uniprotenc $22 \mathrm{~m}$ & 416.848 & 69.703 & 225.292 & 250.250 & 41.326 & 72.286 & 71.939 & 51.734 & 21.791 \\
\hline uniprotenc100m & 720.053 & 139.863 & 411.032 & 327.161 & 99.315 & 110.354 & 109.584 & 112.616 & 48.091 \\
\hline web & 573719 & 254.526 & 1766.090 & 157.571 & 62.971 & 57.815 & 57.206 & 147.893 & 86.664 \\
\hline wiki & 682422 & 76.472 & 161.378 & 55.983 & 45.719 & 77.611 & 78.795 & 26.817 & 27.033 \\
\hline yago & 609.651 & 191.759 & 167.131 & 134.714 & 119.745 & 119.136 & 135.862 & 137.366 & $\mathbf{8 8 . 8 5 4}$ \\
\hline twitter & - & 239.108 & - & 100.933 & 102.923 & 108.730 & 104.698 & 82.212 & 79.285 \\
\hline web-uk & - & 492.230 & - & 216.287 & - & 227.611 & 146.429 & 214.857 & 253.082 \\
\hline citeseerx & 28774.346 & $\overline{4410.155}$ & $\overline{7723.276}$ & 274.382 & 230.318 & 187.795 & 111.329 & $\overline{131.534}$ & $\overline{101.444}$ \\
\hline HostLink & 8693750 & 464.266 & 5433.720 & 185.109 & 264.938 & 119.398 & 138.803 & 184.410 & $\mathbf{1 1 7 . 3 3 9}$ \\
\hline
\end{tabular}

Table 7: Query Time on Large Real Graphs (in millisecond)

graph. twitter is a DAG of the social network graph crawled from twitter.com collected by [6]. web-uk is a DAG of a web graph dataset collected by [2]. We also add two new datasets. dbpedia is the DAG of the knowledge graph DBpedia (dbpedia.org). HostLink is the DAG of the latest 100 million host links extracted from the host link graph data. webarchive.org. uk. All the real graphs are DAGs, and the basic information is given in Table 4. In Table 4, $|V(G)|$ and $|E(G)|$ are the number of vertices and the number of edges, $d_{\text {avg }}$ is the average degree of a graph, and Rratio $(r)$ is the reachability-ratio discussed in Section 4. As shown in Table 4, most of these graphs are tree-like graphs whose $d_{\text {avg }}$ is very closed to 1 . We classify the real graphs into two classes: sparse graphs $\left(d_{\text {avg }}<2\right)$ and dense graphs $\left(d_{\text {avg }} \geq 2\right)$. The 6 dense graphs are citeseerx, patent, go-uniprot, govwild, dbpedia, and HostLink. The R-ratio of reachable pairs is very small, far below $1 \%$ in most cases, which is consistent with our discussion in Section 4. The following 5 datasets, email, LJ, web, twitter, and web-uk, have relatively high R-ratio, because they have some huge-vertices. The neighbors of the huge-vertices nearly cover the entire vertex set of the graphs.

Synthetic datasets: We generate large DAGs with 10 million vertices and an average degree from 2 to 8 using the same graph generation algorithm used in GRAIL [29]. We first randomly create 10 million vertices and do a random ordering for these vertices. Then we randomly pick up two distinct vertices from the whole vertex set and create an edge which points from a lower order vertex to a higher order vertex. By repeating the process, we create the predefined number of edges.

Parameters: The 3 Label $+G$ approaches have parameters to control their index size. The Label $+G$ approaches get better performance when setting the parameters small for sparse graphs and larger for dense graphs. For $I P+$, we set $k=2$ and $h=2$ for sparse graphs and $k=5$ and $h=5$ for dense graphs, and $\mu=100$ by default. By $\mu=100$, a vertex $u$ with $d_{O}(u)$ greater than $\mu$ is considered as a huge-vertex. For Ferrari-G, it is set as $k=2$ for sparse graphs with $s=2$ seeds as an additional index for seed based pruning, and $k=5, s=5$ for dense graphs. Similarly, we set $k=2$ for GRAIL for sparse graphs and $k=5$ for dense graphs. It is worth noting that the exact index sizes consumed by IP+ and Ferrari- $G$ can be bounded but cannot be fully controlled. By setting the similar parameters, the three $I P+$, Ferrari-G, and GRAIL will consume the similar index space.

\subsection{Performance on Large Real Graphs}

We report the index construction time, index size, and query time for large real graphs in Table 5, Table 6 and Table 7. The best results among are highlighted in bold font.

Index construction: Table 5 reports the index construction time. $I P+$ is the fastest in almost all datasets. $I P+$ cannot reach the best results in some other sparse graphs whose $d_{\text {avg }}$ is close to 1 , whereas PWAH8 shows good performance in these sparse graphs. For other sparse graphs and dense graphs, $I P+$ performs the best compared with others. The index construction time of 2-Hop approaches is about 2 to 25 times of that of our approach over the dense graphs, and the index construction of PWAH8 is on average 3 times slower than $I P+$ over the dense graphs. HL fails its index construction in the dense graph patent, whereas TF-Label fails its index construction in the largest graph web-uk. The three versions of GRAIL approaches and Ferrari-G perform significantly slower than $I P+$ in the index construction. For the dense graphs, $I P+$ is twice faster than GRAIL approaches and Ferrari-G. In the large graphs twitter and web-uk, ScaGRAIL fails to compute their reachability backbone.

Index size: Table 6 shows the index size. For the dense graphs patent and go-uniprot, $I P+$ constructs the smallest index, and the index size by $I P+$ is closed to the best result in two other dense graphs citeseerx and govwild. But for other graphs, the Label $+G$ approaches (GRAIL, Ferrari-G, and $I P+$ ) result in an index whose size is larger than those by the three 2-Hop approaches (TF-Label, $H L, D L)$ and PWAH8. This is because GRAIL, Ferrari-G, and IP+ use $O(k)$ labels and the additional index for every vertex no matter how sparse the graph is in practice. In theory, the index size by either GRAIL, Ferrari-G, or IP+ is in linear, whereas the 2-Hop approaches (TF-Label, HL, and DL) and PWAH8 may construct an unacceptably large index in dense graphs as showed in the patent dataset, where the index by 2-Hop approaches is at least 3.5 times larger than the index $I P+$ constructs.

Query time: We randomly generate queries such that every vertex pair will be selected with the same probability. For each dataset, we generate 1 million reachability queries. Table 7 shows the total query time taken to answer all the reachability queries generated. $I P+$ performs the best in 5 sparse graphs among the 13 sparse graphs, and is comparable to the best results in other sparse graphs. IP+ is at least one order of magnitude faster than GRAIL and also much faster than its two improved versions GRAIL ${ }^{*}$ and Sca- 
GRAIL. Ferrari-G shares the similar query performance with $I P+$ in sparse datasets, but significantly slower than $I P+$ for dense graphs. For dense graphs, $I P+$ wins the best in all dense graphs except patent. This is because that nearly a half of vertices in patent have at least 4 out-degree. When it needs to do DFS, it may need to visit more vertices until all branches are pruned or encountering the destination vertex. We believe that IP+ in SCARAB can perform well in the patent dataset, because ScaGRAIL greatly improves the query time in patent compared with GRAIL.

\subsection{Scalability Study on Synthetic Graphs}

We conduct experimental studies on synthetic graphs with different density. Since GRAIL* has better query and index construction performance than original version of GRAIL, we use GRAIL ${ }^{*}$ in the experiments. Fig. 3 shows the experimental results of different approaches on the synthetic graphs generated.

Fig. 3(a) and Fig. 3(b) show the index construction time and index size on graphs with different density. First, except for sparse graphs with the average degree $2, I P+$ always performs the best both on the index construction time and the index size. The index construction time and the index size of $I P+$ only increases marginally while the graph density increases. Second, PWAH8 and 2-Hop approaches (TF-Label, HL, DL) do not show good scalability in large dense graphs. Their index construction time and index size increases exponentially when the average degree of the graph increases linearly. For the dense graphs, their memory usage exceeds the memory size of the system and thus fails to complete the index construction. PWAH8 and HL cannot compute its labels successfully when the average degree $\geq 5$, whereas TF-Label and $D L$ fail their index construction for the graphs whose average degree is $\geq 6$. Third, the index construction time of GRAIL ${ }^{*}$ is on average 2 times larger than that of $I P+$, and the index construction time of Ferrari-G is about 3 times larger than that of $I P+$. The index size of GRAIL ${ }^{*}$ and Ferrari-G is about two times of the index size of $I P+$. In summary, $I P+$ has the best scalability.

We also randomly generate 1 million reachability queries over the synthetic graphs. The total query time to answer all reachability queries generated is shown in Fig. 3(c). The 2-Hop approaches have the better performance in the datasets if they can construc$t$ the index given the time and space limited. The query time of $I P+$ is comparable to the 2-Hop approaches (TF-Label, HL, DL) in graphs with average degree $\leq 3 . I P+$ is on average twice faster than $P W A H 8$. Only GRAIL ${ }^{*}$, Ferrari-G, and $I P+$ can answer the query in dense graphs with average degree $\geq 6$. And the query time of $I P+$ is about a half of the query time of GRAIL ${ }^{*}$ and Ferrari-G in all graphs. For answering a single reachability query in the densest graph with an average degree 8 , the query time of $I P+$ is on average 0.75 millisecond, whereas GRAIL* and Ferrari-G need more than 1 millisecond. $I P+$ is the best approach among all approaches that can scale to large dense graphs.

\subsection{IP label $V S$ interval label}

We show that only IP+, GRAIL*, and Ferrari-G are scalable to handle large dense graphs. Unlike GRAIL and Ferrari-G, IP uses a 2-Hop-like label, instead of interval labeling. To further study the query time of IP label vs interval label, we compare IP label, GRAIL label, and Ferrari-G index by excluding any additional index and any query optimization techniques. For fairness, we use similar index sizes for the three approaches. We conduct two testings.

First, we test the same 1 million random queries generated over the synthetic graph with an average degree 3 we used for the scalability testing. Index sizes for the three approaches are: $776 \mathrm{MB}$

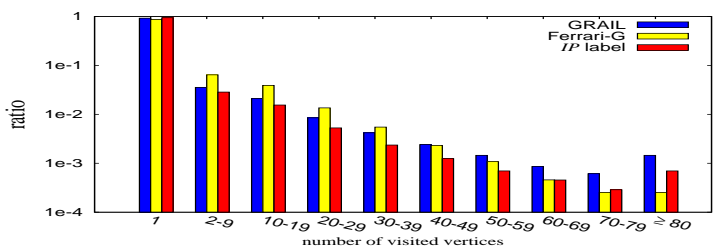

Figure 4: Distribution of the number of vertices visited

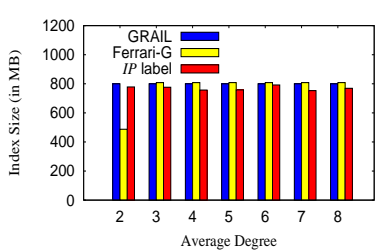

(a) Index Size

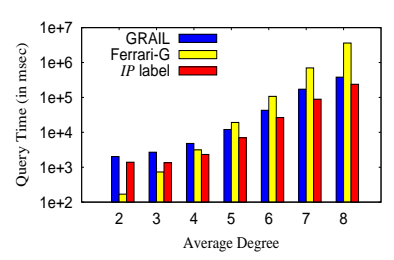

(b) Query Time
Figure 5: Equal Query Workload

for IP labels, 800MB for GRAIL labels, and 809MB for Ferrari-G index. We measure how many vertices they visit in answering the reachability queries generated. Fig. 4 shows the distribution of the number of visited vertices. $94.5 \%$ of the queries can be answered directly using IP labels while $92.3 \%$ of the queries can be answered directly using GRAIL labels and $87.3 \%$ of the queries can be answered directly using Ferrari-G index. It shows that $I P$ label has high potential to answer without the needs of DFS. For the reachability queries that cannot be answered directly using labels, we can see that IP label also performs significantly better than GRAIL and Ferrari-G. Compared with the two interval labeling approaches, IP label is less likely to visit many vertices before finally answering a reachability query. The percentages of visiting more than 30 vertices using IP, GRAIL, and Ferrari-G, are $0.57 \%, 1.10 \%, 0.99 \%$. The percentage of IP is about a half of either GRAIL or Ferrari-G. For reachability queries that need to visit more than 70 vertices, the ratio of IP label is larger than the ratio of Ferrari- $G$ index but the ratio of IP label is still below $0.1 \%$. The results shown are consistent with the experiments in which IP label usually has better query performance than GRAIL and Ferrari-G.

Second, we test equal query workload with 500,000 reachable queries and 500,000 non-reachable queries sampled from TC over the same 7 synthetic graphs we used for the scalability testing (average degree is from 2 to 8 ). The index size and the query time are shown in Fig. 5. Ferrari-G performs well when the average degree is 2 , where the index size cannot be larger by controlling the parameters provided in Ferrari-G. Overall, IP performs the best.

\subsection{The IP+ Label}

Here, first, we report how the 3 parameters, $k, h$, and $\mu$, affect the query performance of $I P+$. Second, we report the effectiveness of the three labels used in IP+, IP label, Level label, and HV-Label. We conducted extensive testings by 1 million random queries generated. We report the results using the real graph govwild whose average degree is 2.95. As default, $k=5, h=5$, and $\mu=100$.

Fig. 6(a) shows that a larger $k$ can improve the query time, but it does not improve the query time when $k$ is too large. Fig. 6(b) shows that $H V$-Label helps. A small $h$ is sufficient because there is a few huge-vertices in a real graph. Fig. 6(c) shows that a smaller $\mu$ is sufficient to improve the query time. It confirms that $\mu=$ 100 is reasonable. When $\mu$ becomes smaller, many vertices will be marked as a huge-vertex, which increase the index size. Fig. 6(d) shows the effectiveness of the labels used in $I P+$. We compare IP+ with IP, IP plus Level, and IP plus HV-Label. With IP label only, it 


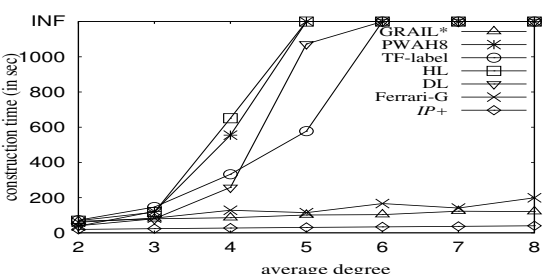

(a) Construction Time

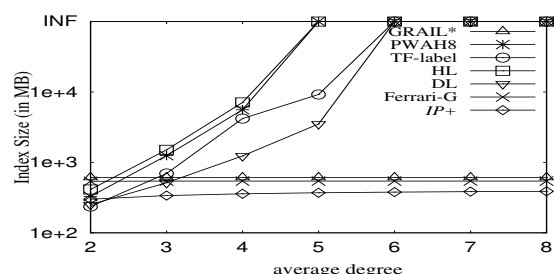

(b) Index Size

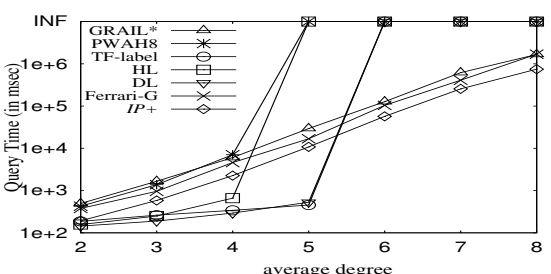

(c) Query Time

Figure 3: Query Synthetic graphs with average degree 2 to 8

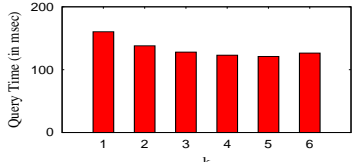

(a) Vary $k$

(c) Vary $\mu$

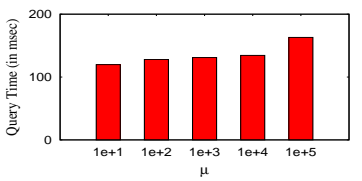

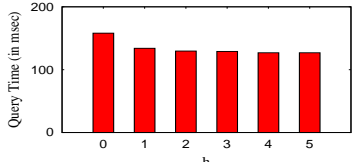

(b) Vary $h$

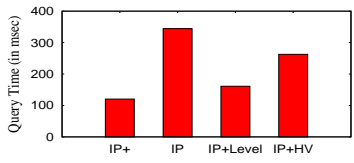

(d) Label Combinations
Figure 6: $I P+$

needs 344 millisecond to answer all queries. Level label and $H V$ Label improve the query time effectively.

\section{CONCLUSIONS}

In this paper, we propose a new IP labeling approach, which is the first one to explore the randomness to answer reachability queries. Like the up-to-date Label-Only approaches (TF-Label, $\mathrm{H}$ $L$, and $D L), I P$ uses two sets of vertices. Unlike TF-Label, HL, and $D L, I P$ uses set-containment instead of set-intersection. The fundamental difference behind answering $\operatorname{Reach}(u, v)$ is as follows. TF-Label, HL, and DL ensure $u \rightsquigarrow v$ using the labels, whereas IP is on the opposite aiming at $u \not \sim v$ by finding at least one vertex in one set that is not contained in the other. IP is effective given the small reachability-ratio for all large graphs. IP outperforms the up-to-date Label $+G$ approaches, GRAIL and Ferrari. The randomness by independent permutation used in IP opens a new direction to study new labeling approaches, in order to further improve the query processing time while minimizing the index construction time/space.

ACKNOWLEDGEMENTS: The work was supported by the grant of the Research Grants Council of the Hong Kong SAR, China, No. 418512.

\section{REFERENCES}

[1] R. Agrawal, A. Borgida, and H. V. Jagadish. Efficient management of transitive relationships in large data and knowledge bases. In Proc. of SIGMOD’89, 1989.

[2] P. Boldi, M. Santini, and S. Vigna. A large time-aware web graph. SIGIR Forum, 42(2), 2008.

[3] A. Broder. On the resemblance and containment of documents. In Proc. of SEQUENCES'97, 1997.

[4] A. Z. Broder, M. Charikar, A. M. Frieze, and M. Mitzenmacher. Min-wise independent permutations. In Proc. of STOC'98, 1998.

[5] J. Cai and C. K. Poon. Path-hop: efficiently indexing large graphs for reachability queries. In Proc. of CIKM'10, 2010.

[6] M. Cha, H. Haddadi, F. Benevenuto, and P. K. Gummadi. Measuring user influence in twitter: The million follower fallacy. In Proc. of ICWSM'10, 2010.

[7] L. Chen, A. Gupta, and M. E. Kurul. Stack-based algorithms for pattern matching on dags. In Proc. of VLDB'05, 2005.
[8] Y. Chen and Y. Chen. An efficient algorithm for answering graph reachability queries. In Proc. of ICDE'08, 2008.

[9] Y. Chen and Y. Chen. Decomposing dags into spanning trees: A new way to compress transitive closures. In Proc. of ICDE'11, 2011.

[10] J. Cheng, S. Huang, H. Wu, and A. W.-C. Fu. Tf-label: a topological-folding labeling scheme for reachability querying in a large graph. In Proc. of SIGMOD'13, 2013.

[11] J. Cheng, J. X. Yu, X. Lin, H. Wang, and P. S. Yu. Fast computation of reachability labeling for large graphs. In Proc. of EDBT'06, 2006.

[12] J. Cheng, J. X. Yu, X. Lin, H. Wang, and P. S. Yu. Fast computing reachability labelings for large graphs with high compression rate. In Proc. of EDBT'08, 2008.

[13] E. Cohen, E. Halperin, H. Kaplan, and U. Zwick. Reachability and distance queries via 2-hop labels. In Proc. of SODA'02, 2002.

[14] M. Faloutsos, P. Faloutsos, and C. Faloutsos. On power-law relationships of the internet topology. ACM SIGCOMM Computer Communication Review, 29(4), 1999.

[15] R. A. Fisher, F. Yates, et al. Statistical tables for biological, agricultural and medical research. Oliver and Boyd, Edinburgh, 3rd edition, 1949.

[16] H. V. Jagadish. A compression technique to materialize transitive closure. ACM Trans. Database Syst., 15(4), 1990.

[17] R. Jin, N. Ruan, S. Dey, and J. X. Yu. Scarab: scaling reachability computation on large graphs. In Proc. of SIGMOD'12, 2012.

[18] R. Jin, N. Ruan, Y. Xiang, and H. Wang. Path-tree: An efficient reachability indexing scheme for large directed graphs. ACM Trans. Database Syst., 36(1), 2011.

[19] R. Jin and G. Wang. Simple, fast, and scalable reachability oracle. $P V L D B, 6(14), 2013$.

[20] R. Jin, Y. Xiang, N. Ruan, and D. Fuhry. 3-HOP: A high-compression indexing scheme for reachability query. In Proc. of SIGMOD’09, 2009.

[21] R. Jin, Y. Xiang, N. Ruan, and H. Wang. Efficiently answering reachability queries on very large directed graphs. In Proc. of SIGMOD'08, 2008.

[22] D. E. Knuth. The art of computer programming, volume 2: seminumerical algorithms, 1981.

[23] R. Schenkel, A. Theobald, and G. Weikum. Hopi: An efficient connection index for complex XML document collections. In Proc. of EDBT'04, 2004.

[24] S. Seufert, A. Anand, S. J. Bedathur, and G. Weikum. Ferrari: Flexible and efficient reachability range assignment for graph indexing. In Proc. of ICDE'13, 2013.

[25] K. Simon. An improved algorithm for transitive closure on acyclic digraphs. Theor. Comput. Sci., 58(1-3):325-346, 1988.

[26] S. TrißI and U. Leser. Fast and practical indexing and querying of very large graphs. In Proc. of SIGMOD’07, 2007.

[27] S. J. van Schaik and O. de Moor. A memory efficient reachability data structure through bit vector compression. In Proc. of SIGMOD'11, 2011

[28] H. Wang, H. He, J. Yang, P. S. Yu, and J. X. Yu. Dual labeling: Answering graph reachability queries in constant time. In Proc. of ICDE'06, 2006.

[29] H. Yildirim, V. Chaoji, and M. J. Zaki. Grail: Scalable reachability index for large graphs. PVLDB, 3(1), 2010.

[30] H. Yildirim, V. Chaoji, and M. J. Zaki. Grail: a scalable index for reachability queries in very large graphs. VLDB Journal, 21(4), 2012.

[31] J. X. Yu and J. Cheng. Graph reachability queries: A survey. In Managing and Mining Graph Data, pages 181-215. Springer, 2010. 